\title{
Sequential Resource Allocation for Nonprofit Operations
}

\author{
Robert W. Lien, Seyed M.R. Iravani and Karen R. Smilowitz \\ Department of Industrial Engineering and Management Sciences \\ Northwestern University, Evanston, IL 60208, USA
}

\begin{abstract}
This paper studies a sequential resource allocation problem motivated by distribution operations of a nonprofit organization. The alternate objectives that arise in nonprofit, as opposed to commercial, operations lead to new variations on traditional problems in operations research and inventory management. Specifically, we consider the problem of distributing a scarce resource to meet sequentially observed customer demand. In a commercial setting, the amount distributed to each customer is determined to maximize profit; however, this objective may lead to inequitable distributions among customers. Our work in a nonprofit setting solves the sequential resource allocation problem with an objective function aimed at equitable and sustainable service. We define service in terms of fill rate (the ratio of the allocated amount to observed demand) and develop an objective function to maximize the expected minimum fill rate among customers. Through a dynamic programming framework, we characterize the structure of the optimal allocation policy for a given sequence of customers. In addition, we address customer visitation sequencing by identifying properties to consider in sequencing decisions to optimize the objective. For both inventory allocation and customer sequencing decisions, we develop heuristic methods which yield near-optimal solutions.
\end{abstract}

Subject classifications: Inventory/production: sequential resource allocation. Transportation. Area of review: Manufacturing, Service and Supply Chain Operations

\section{Introduction and Motivation}

While America has the strongest national economy with a GDP of 13.2 trillion dollars in 2006, 12.3\% of its population was below the poverty line in that same year. Based on the most recent hunger study from America's Second Harvest, thirty-six million Americans suffer from hunger. Twenty-five million of these Americans rely on America's Second Harvest and their network of pantries, shelters and soup kitchens for food. The largest suppliers to these agencies are regional and local food banks. Food banks are large distribution centers which collect, store and distribute food. Much of this food is donated by sources of surplus food such as supermarkets and grocery chains. According to the U.S. Department of Agriculture, 100 billion pounds of food are wasted each year in the United States. The goal of American's Second Harvest (ASH) and the agencies in their network is to match surplus food with those in need. This matching is a large-scale distribution and inventory management problem that occurs each day at thousands of nonprofit agencies across the country. Much research has been conducted on related supply chain problems in commercial settings where the goal of such systems 
is either to maximize profit or minimize cost. Little work, however, has been conducted in nonprofit applications. In such settings, the objectives are often more difficult to quantify since issues such as equity and sustainability must be considered, yet efficient operations are still crucial.

Several decades of research in commercial supply chains have resulted in papers that model a wide range of inventory management decisions. In general, these models are designed to maximize a firm's profit and provide useful insight in inventory management. Many firms assign a substantial budget to $R \& D$ activities to improve the profitability of their organization; nonprofit organizations rarely have funds for such activities. Unfortunately, many models developed for commercial settings are not applicable to nonprofit organizations. It is essential to provide answers to inventory management questions that occur in nonprofit organizations such as food banks, where the main objectives are to ensure that food is distributed fairly and waste is minimized.

The Greater Chicago Food Depository (GCFD) is an active ASH member. According to a 2005 study by the GCFD and ASH, 500,000 people in the Chicago region are served by the GCFD each year. Lisa Koch, GCFD's former director of public policy, notes "more and more people are in need of supplemental and emergency food, and we need continued support to shrink the gap between those who need help and our means to provide it." We have worked with the GCFD to address this gap, focusing on their Food Rescue Program (FRP). The FRP distributes perishable food from donors (e.g. supermarkets and restaurants) to agencies (e.g. shelters and soup kitchens). Over 80 donors and 100 agencies participate in the FRP, which moves over 4 million pounds of food annually.

The FRP operates 5 truck routes, each visiting, on average, 8 donors and 4-5 agencies daily. A sample route is shown in Figure 1. Stop A represents the GCFD warehouse where trucks are housed (referred to as the depot in this paper), stops represented by B through E are donors and stops $\mathrm{F}$ through I are agencies. Due to operating schedules of the donor sites, routes first visit all donors before visiting agencies. Routes are scheduled weeks in advance and remain fairly regular to facilitate driver familiarity. The frequency of visits to a location over the course of a month depends on the supply (for donors) and food need (for agencies).

Donation amounts are unknown until observed upon the driver's arrival. All donated food is accepted and loaded; there is typically ample truck capacity for the donated food. Food demand depends on available storage and budget at the agencies; agencies are charged 4 cents per pound. Under current operations, because of lack of personnel at agencies and timing of delivery operations, agency requests are revealed only upon arrival. The allocation of food to agencies is left to the discretion of 


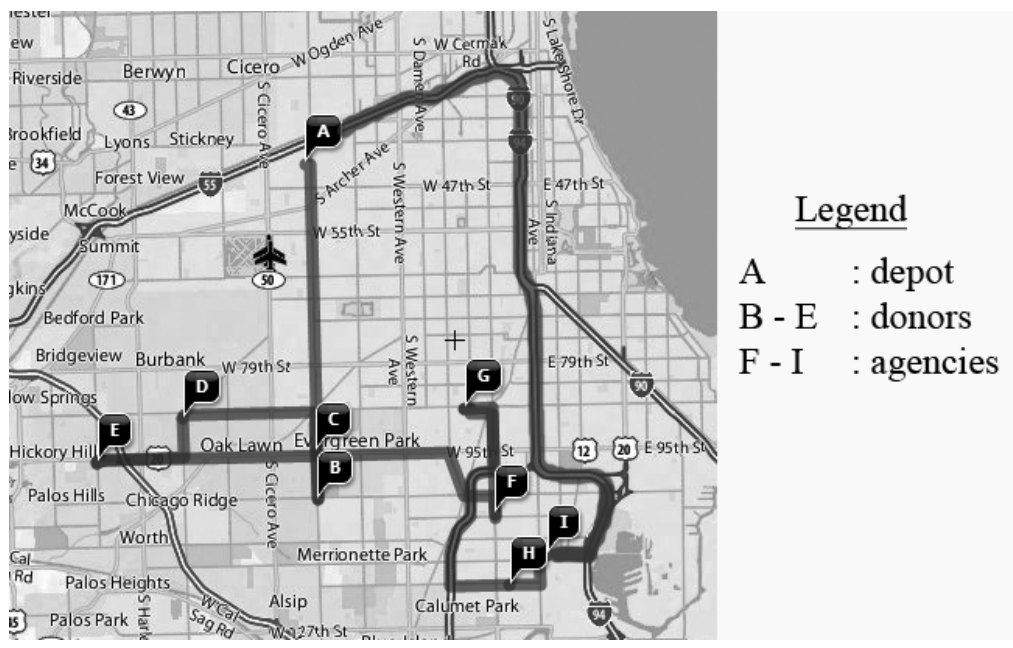

Figure 1: Sample Food Rescue Program route in Greater Chicago area

the driver who tries to satisfy an agency's demand while reserving supply for the remaining agencies on the route.

Given a set of donors and agencies, the driver's decision can be modeled as a Sequential Resource Allocation problem (SRA), where the resource in this case is food and allocation decisions are made as demands are observed sequentially at each node (agency). The SRA with profit based objectives (SRAp) of maximizing revenue or minimizing costs has been studied by Bassok and Ernst (1995), Kumar et al. (1995) and Berman and Larson (2001). Although cost-efficient operations remain desirable in nonprofit operations, focusing purely on cost can lead to inequitable solutions. Our research considers the SRA with an objective that considers equity (SRA-e).

In designing policies for the SRA-e, the following allocation and sequencing issues are addressed.

1. What is an appropriate objective to determine resource allocation to ensure equity?

2. Given an objective, what allocation policy should be implemented?

3. How should nodes be sequenced within a route to optimize the given objective?

In this paper, we answer these questions to develop operating policies for the SRA-e. In addition to the Food Rescue Program, the SRA-e model is applicable in humanitarian and disaster relief efforts, where the resources are scarce relief supplies. In Section 2, we review existing literature on similar resource allocation problems for the commercial sector, as well as related problems with equity and other nonprofit objectives. In Section 3, we develop the objective function for the SRA-e and describe the dynamic programming framework used to solve the SRA-e. We address allocation policies for a fixed route in Section 4 and the sequencing of nodes along a route in Section 5 . In both 
sections we derive analytical results which provide insights in developing effective heuristic methods. In Section 6, we combine the heuristic allocation and sequencing methods and compare the results against optimal operating policies. In Section 7, we present a numerical study to assess the value of demand information, and in Section 8 we study the expected waste under the optimal allocation policy. Lastly, in Section 9 we conclude the paper and discuss research extensions.

\section{$2 \quad$ Literature Review}

Several papers have addressed allocation for the SRA-p on fixed delivery routes in commercial operations. Bassok and Ernst (1995) examine a system where demand is observed sequentially upon arrival at a customer. Given varying profit margins by customer, the driver may be willing to trade a sure profit for potentially higher profit at a customer later in the route. They show that the revenue maximizing allocation policy at each customer is a threshold policy independent of customer demand. Kumar et al. (1995) evaluate static and dynamic allocation policies in delivery routes with long travel times between customers. Static policies are predetermined allocation amounts that do not change once customers are visited, while dynamic policies adjust as demand is observed. In their model, the driver has preliminary inventory information for customers, though actual inventory levels change during vehicle travel. Berman and Larson (2001) study a similar problem in the allocation of industrial gases. They apply an incremental cost structure to quantify the value of timely delivery and product fulfillment. These papers optimize profit/cost-based objectives, where the profit/cost from serving a customer is directly added in the objective function.

Some research on nonprofit applications have similar additive objective functions that maximize utility/service or minimize costs, see Chou et al. (2008), Wong and Meyer (1993) and Johnson et al. (2005). However, the majority of operations research literature in nonprofit settings incorporate equity as an objective. See Pollock et al. (1994) for a review of operations research work in the public sector, with a focus on government activities; and Johnson and Smilowitz (2007) for an overview of research in community-based nonprofit operations. Gass (1994) discusses the differences between private and public applications of operations research and notes the particular emphasis on equity in nonprofit operations. Further, Savas (1978) identifies efficiency, effectiveness and equity as key performance measures in nonprofit settings.

The literature has considered different methods to measure and incorporate equity. In Mandell (1991), equity is measured with the Gini coefficient, and is incorporated as an objective in a multi- 
objective mathematical programming model. This work considers the tradeoff between effectiveness and equity as applied to the allocation of books to libraries. Campbell et al. (2008) explore two objective functions for the local distribution of supplies in a relief effort. To minimize disparity in response times to recipients, the authors consider the objectives of minimizing the arrival of supplies to the last recipient and minimizing the average arrival of supplies. They show that the choice of objective function can have significant impact on solutions. Swaminathan (2003) considers the application of allocating scarce drugs to clinics and hospitals. In this work, tradeoffs between equity, effectiveness and efficiency are modeled in an multi-objective mathematical program. In these papers, static decision models, such as mathematical programs, are appropriate since decisions are made at a single epoch; in our work, however, each node represents a decision epoch.

Our paper contributes to the literature by introducing a sequential allocation problem with a nonprofit objective. By necessity, we employ a measure of equity and an objective function which are different from what has been done in the literature. Because of the mathematical structure of the objective function, the solution methods and results from commercial sequential allocation research are not directly applicable. In this paper, we develop a new model and solution approaches to address the unique problem motivated by the FRP.

\section{Sequential Allocation Model}

In Section 3.1, we develop the objective function for the SRA-e. In Section 3.2, we present the dynamic programming formulation to solve the model with analytical results for optimal values.

\subsection{Objective Function Development}

Consider the simple FRP example presented in Figure 2, in which the supply is collected from one donor (source) and distributed to two agencies (nodes). Since all donations are accepted and loaded, our model aggregates donors into one supply source without loss of generality. The first decision epoch for the SRA-e model occurs at node 1 , which is the first agency visited on the route. Demand $\left(D_{i}\right)$ at node $i$ follows the discrete probability distribution presented in the figure. Let $d_{i}$ denote the observed demand at node $i$ and $x_{i}$ denote the amount allocated to $i$. We define the fill rate at node $i$ as $\beta_{i}=\frac{x_{i}}{d_{i}}$. The amount of initial supply $\left(s_{0}\right)$ is a known parameter of the model. Let $s_{i}$ denote the units of supply available upon arrival at node $i$; therefore, $s_{1}=s_{0}$ and $s_{i}=s_{i-1}-x_{i-1}$.

In the example in Figure 2, $s_{0}=130$ units of supply. Upon arrival at node 1, allocation is 


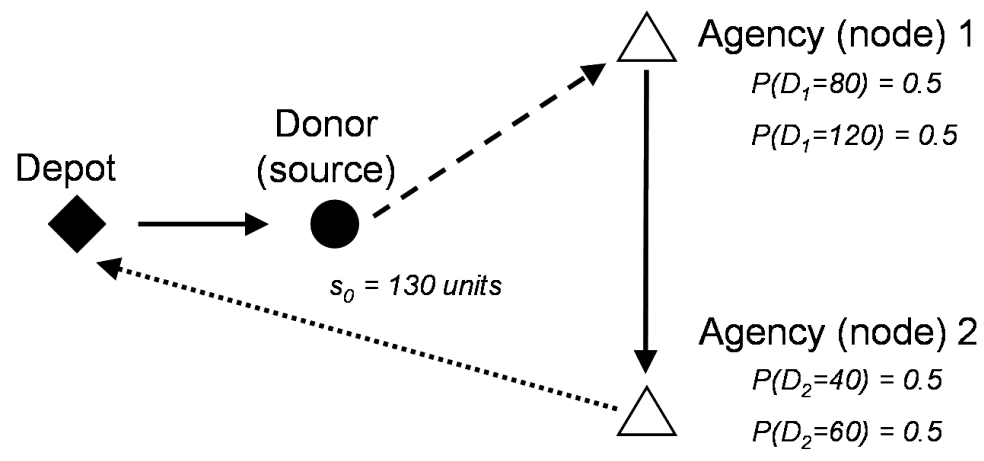

Figure 2: Example of a food distribution route

determined to optimize a chosen objective function. For example, if the objective is to minimize expected waste, it is optimal to satisfy all of the observed demand at node 1 . Therefore, if $d_{1}=80$, then $x_{1}=80$, and if $d_{1}=120$, then $x_{1}=120$. As a result, the available supply upon arrival at node 2 is $s_{2}=50$ with probability 0.5 and $s_{2}=10$ with probability 0.5 . The allocation at node 2 is $x_{2}=\min \left\{d_{2}, s_{2}\right\}$; the demand at node 2 is fully satisfied if possible. Considering all possible demand realizations for the nodes, under the objective of minimizing waste, the expected waste is 2.5 units, which is $2 \%$ of the initial supply. The expected fill rates at nodes 1 and 2 are $E\left[\beta_{1}\right]=100 \%$ and $E\left[\beta_{2}\right]=56 \%$, respectively.

The above solution minimizes expected waste (and maximizes expected distribution), yet there is a $44 \%$ difference between the fill rates of the agencies. The goal of maintaining equity among agencies is not achieved. To consider equity while maintaining a high level of distribution, we consider the objective of maximizing the expected minimum fill rate (i.e. $\max \left\{\mathbb{E}\left[\min \left\{\beta_{1}, \beta_{2}\right\}\right]\right\}$ ). Note that the fill rates of all nodes are bounded below by the minimum fill rate and above by 1 . Increasing the minimum fill rate among all nodes improves overall distribution by increasing the fill rates of all agencies and improves equity by reducing the difference between fill rates.

We show in Section 3.2 that using dynamic programming under this objective the optimal allocation policy is as follows: If $d_{1}=80$, then $x_{1}=75$, and if $d_{1}=120$, then $x_{1}=87$. As a result, $s_{2}=55$ with probability 0.5 and $s_{2}=43$ with probability 0.5 . As with the previous objective, $x_{2}=\min \left\{d_{2}, s_{2}\right\}$. Considering all four possible demand realizations, the expected fill rates at nodes 1 and 2 are $E\left[\beta_{1}\right]=$ $83 \%$ and $E\left[\beta_{2}\right]=91 \%$, respectively. The expected waste is 4.5 units, which is $3.5 \%$ of the initial and only $1.5 \%$ more than the previous objective.

We focus on maximizing the expected minimum fill rate, which is consistent with the goals of the GCFD: 
- Equity - This objective ensures that agencies are treated equally by serving an equitable portion of each agency's needs.

- Sustainability of each agency - Raising overall distribution ensures that all participant agencies benefit from the food distribution program. Agencies are the most important means in helping the hungry; sustaining them is a primary concern for the food bank.

- Publicity statement - The food bank can improve and publicize the level of service to all agencies in the program. The decision process is more transparent, which can improve cooperation among donors, agencies and the food bank. Good publicity is crucial for private funding and community support.

\subsection{Dynamic Programming Model}

For a SRA-e consisting of $N$ nodes in the fixed sequence $1 \rightarrow 2 \rightarrow \cdots \rightarrow N$, a dynamic programming model can be generalized as follows. At each node, an allocation decision is made given the current supply, observed demand at the node, minimum fill rate among nodes already visited, and demand distributions of nodes yet to be visited. Suppose that nodes 1 to $i-1$ have been visited and the resource allocation decisions resulted in fill rates $\beta_{1}, \beta_{2}, \ldots, \beta_{i-1}$. Let $\beta_{\min }^{i-1}=\min \left\{\beta_{1}, \beta_{2}, \ldots, \beta_{i-1}\right\}$. To obtain $x_{i}^{*}$, the optimal allocation policy at node $i$, we solve the following optimality equation:

$$
Z^{(i)}\left(s_{i}, \beta_{\min }^{i-1}, d_{i}\right)=\max _{x_{i}} \mathbb{E}_{D_{i+1}}\left[1 \wedge \beta_{\min }^{i-1} \wedge \frac{x_{i}}{d_{i}} \wedge Z^{(i+1)}\left(s_{i}-x_{i}, \beta_{\min }^{i}, d_{i+1}\right)\right]
$$

where $a \wedge b=\min \{a, b\}$, and $Z^{(i)}\left(s_{i}, \beta_{\text {min }}^{i-1}, d_{i}\right)$ is the optimal expected minimum fill rate for the sequence $1 \rightarrow 2 \rightarrow \cdots \rightarrow N$, given that nodes 1 to $i-1$ have been visited with a minimum fill rate $\beta_{\text {min }}^{i-1}$, and supply $s_{i}$ is available upon arrival at node $i$ which has demand $d_{i}$. For node $N$, the last node in the sequence, the allocation is the minimum of demand or supply: $x_{N}^{*}=s_{N} \wedge d_{N}$, and

$$
Z^{(N)}\left(s_{N}, \beta_{\min }^{N-1}, d_{N}\right)=1 \wedge \beta_{\min }^{N-1} \wedge \frac{s_{N} \wedge d_{N}}{d_{N}} .
$$

To find the optimal resource allocation at all stages, one needs to solve for $Z^{(1)}\left(s_{1}, \beta_{m i n}^{1}, d_{1}\right)$, where $\beta_{\min }^{1}=1$. The optimal expected minimum fill rate can be obtained as follows:

$$
\mathcal{Z}\left(s_{1}\right)=\mathbb{E}_{D_{1}}\left[Z^{(1)}\left(s_{1}, 1, d_{1}\right)\right]
$$

To simplify notation, let $\mathcal{B}_{D_{i+1}}\left(x_{i} \mid s_{i}, \beta_{\text {min }}^{i-1}, d_{i}\right)$ represent the expected minimum fill rate for allocation $x_{i}$ at node $i$.

$$
\mathcal{B}_{D_{i+1}}\left(x_{i} \mid s_{i}, \beta_{m i n}^{i-1}, d_{i}\right)=\mathbb{E}_{D_{i+1}}\left[1 \wedge \beta_{m i n}^{i-1} \wedge \frac{x_{i}}{d_{i}} \wedge Z^{(i+1)}\left(s_{i}-x_{i}, \beta_{m i n}^{i}, d_{i+1}\right)\right]
$$


Equation (1) can be rewritten to maximize $\mathcal{B}_{D_{i+1}}(\cdot)$,

$$
Z^{(i)}\left(s_{i}, \beta_{\min }^{i-1}, d_{i}\right)=\max _{x_{i}} \mathcal{B}_{D_{i+1}}\left(x_{i} \mid s_{i}, \beta_{\text {min }}^{i-1}, d_{i}\right)
$$

Lemmas 1 and 2 characterize the behavior of the optimality equation (5) with respect to changes in $d_{i}$ and $s_{i}$. The proofs of all claims in this paper are presented in On-Line Appendix B.

Lemma 1. The family of functions $\mathcal{B}_{D_{i+1}}\left(x_{i} \mid s_{i}, \beta_{\text {min }}^{i-1}, d_{i}\right)$ is concave with respect to the allocation decision, $x_{i}$, and the supply, $s_{i}$.

Lemma 2. The optimal value $Z^{(i)}\left(s_{i}, \beta_{m i n}^{i-1}, d_{i}\right)$ :

(i) is non-decreasing with respect to supply, $s_{i}$; i.e., if $s_{i}^{A}>s_{i}^{B}$, then $Z^{(i)}\left(s_{i}^{A}, \beta_{m i n}^{i-1}, d_{i}\right) \geq Z^{(i)}\left(s_{i}^{B}, \beta_{m i n}^{i-1}, d_{i}\right)$.

(ii) is non-increasing with respect to demand, $d_{i}$; i.e., if $d_{i}^{A}<d_{i}^{B}$, then $Z^{(i)}\left(s_{i}, \beta_{m i n}^{i-1}, d_{i}^{A}\right) \geq Z^{(i)}\left(s_{i}, \beta_{m i n}^{i-1}, d_{i}^{B}\right)$.

Result $(i)$ of Lemma 2 is intuitive: the supply at any point in the route constrains the allocation amount. As $s_{i}$ increases, the optimal expected minimum fill rate cannot decrease. Since fill rates are bounded by 1 , then with Lemma 1 we conclude that the optimal value is an increasing concave function of $s_{i}$ and is bounded by 1 ; i.e., $Z^{(i)}\left(s_{i}, \beta_{m i n}^{i-1}, d_{i}\right) \rightarrow 1$ as $s_{i} \rightarrow \infty$. The intuition behind $(i i)$ is slightly different. As demand increases, a larger allocation is necessary to maintain a fill rate level, which reduces the amount available for nodes yet to be visited; thus, as demand increases, the optimal expected minimum fill rate cannot increase.

\section{Resource Allocation Policies}

In this section we study resource allocation policies. In Section 4.1 we characterize the structure of the optimal allocation policy and its corresponding optimal values. Based on the insights from this analysis we construct a heuristic allocation policy, presented in Section 4.2.

\subsection{Optimal Allocation Policy}

To gain insights into the structure of the optimal allocation policy, we first study a case with two nodes. We later show that the insights from the two-node SRA-e also hold for systems with more than two nodes. For a two-node problem, the optimality equation (1) is:

$$
Z^{(1)}\left(s_{1}, 1, d_{1}\right)=\max _{x_{1}} \mathcal{B}_{D_{2}}\left(x_{1} \mid s_{1}, 1, d_{1}\right)=\max _{x_{1}} \mathbb{E}_{D_{2}}\left[1 \wedge \frac{x_{1}}{d_{1}} \wedge \frac{s_{1}-x_{1}}{d_{2}}\right]
$$


Since the second node is the last node visited, its allocation is $\left(s_{1}-x_{1}\right) \wedge d_{2}$, which is the minimum of available supply and observed demand. The objective function value of the entire sequence is:

$$
\mathcal{Z}\left(s_{1}\right)=\mathbb{E}_{D_{1}}\left[Z^{(1)}\left(s_{1}, 1, d_{1}\right)\right]
$$

The structure of the optimal allocation policy, $x_{1}^{*}$, with respect to $d_{1}$ and $s_{1}$ is illustrated in Figure 3 for a two-node example where both nodes observe Normally distributed demand with $\mu=60$ and $\sigma=18$. The initial supply, $s_{1}$, ranges from 10 to 250 , and the observed demand at node $1, d_{1}$, ranges from 13 to 103 .

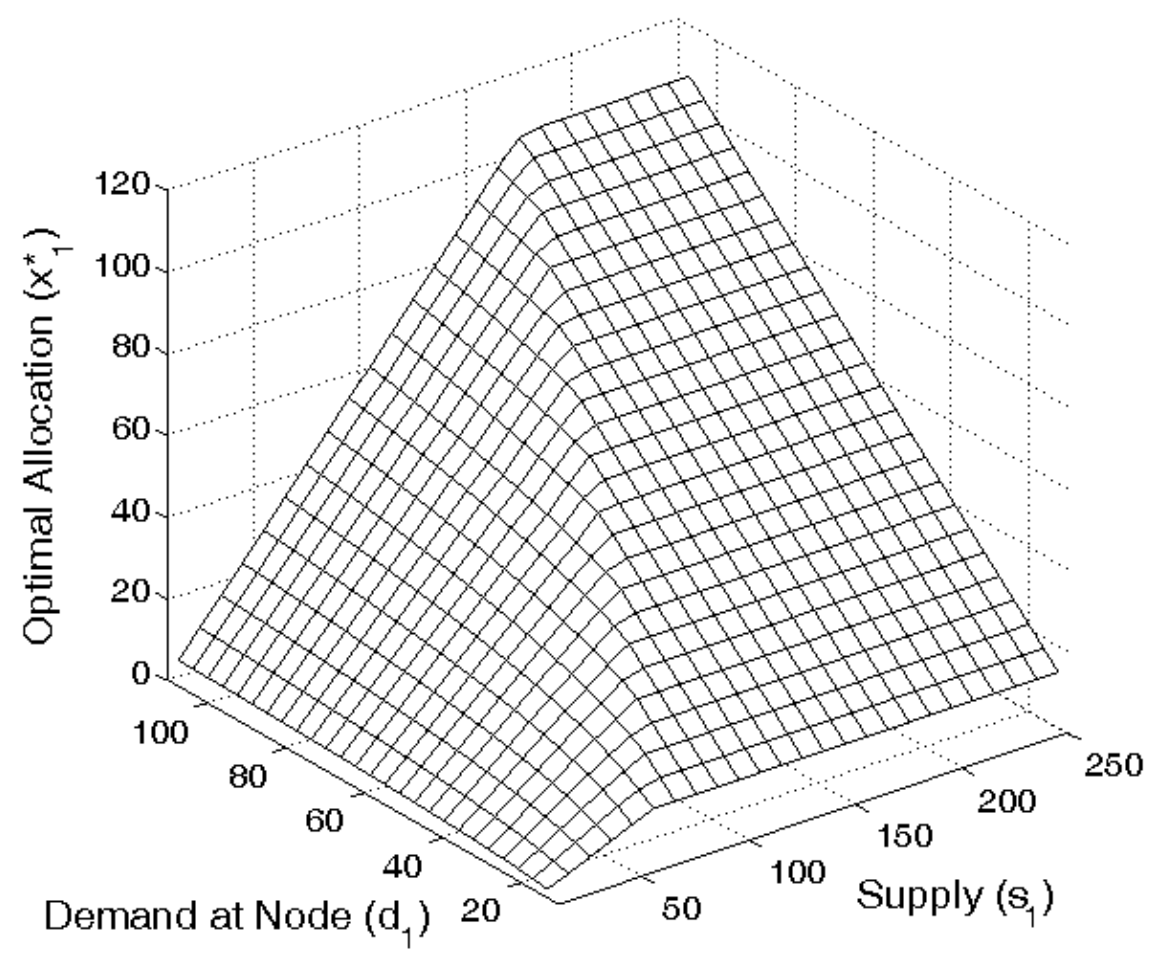

Figure 3: Optimal resource allocation policy $x_{1}^{*}$ as a function of $d_{1}$ and $s_{1}$

From Figure 3, we see that $x_{1}^{*}$ is non-decreasing in $d_{1}$ for any value of $s_{1}$. For each value of $s_{1}, x_{1}^{*}$ follows a piecewise function with respect to $d_{1}$. The piecewise nonlinear structure has the following property: there exists a value of demand, $T_{d}\left(s_{1}\right)$, such that if $d_{1} \leq T_{d}\left(s_{1}\right)$ then $x_{1}^{*}=d_{1}$. For any value of $d_{1} x_{1}^{*}$ is piecewise linear function of $s_{1}$. For each value of $d_{1}$, there exists a value of supply, $T_{s}\left(d_{1}\right)$, such that if $s_{1}<T_{s}\left(d_{1}\right)$, then $x_{1}^{*}$ is a linear function of $s_{1}$; otherwise $x_{1}^{*}=d_{1}$.

We prove these observations in Theorems 1 and 2. Theorem 1 characterizes the threshold structure of the optimal solution with respect to demand at node 1, and Theorem 2 presents the sensitivity of 
the optimal solution and objective value with respect to supply.

Theorem 1. (Structure of Optimal Resource Allocation Policy) In a two-node SRA-e problem, for a given supply $s_{1}$, the optimal allocation $x_{1}^{*}$ is a piecewise function of $d_{1}$. Specifically, there exists a threshold, $T_{d}\left(s_{1}\right)$, such that:

(i) if $d_{1} \leq T_{d}\left(s_{1}\right)$, then the optimal allocation, $x_{1}^{*}=d_{1}$.

(ii) if $d_{1}>T_{d}\left(s_{1}\right)$, then the optimal allocation, $x_{1}^{*}=H_{1}\left(s_{1}, d_{1}\right)$, where $H_{1}\left(s_{1}, d_{1}\right)<d_{1}$.

(iii) the threshold value, $T_{d}\left(s_{1}\right)$, is non-decreasing in $s_{1}$, and $H_{1}\left(s_{1}, d_{1}\right)$ is a strictly increasing concave function of $d_{1}$.
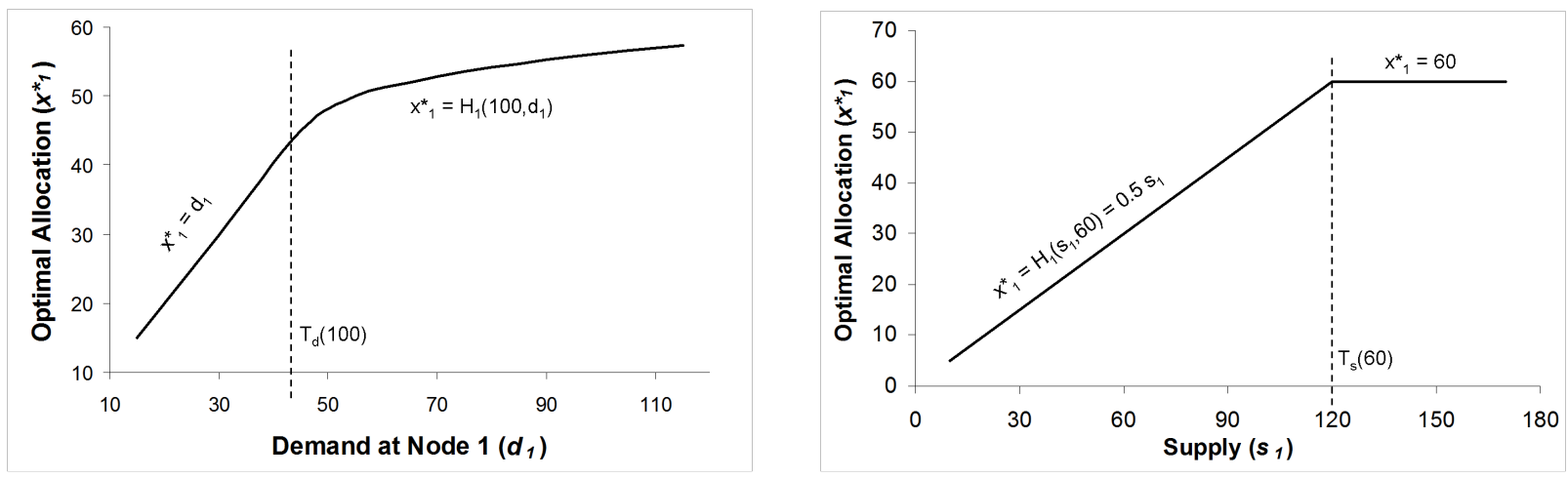

Figure 4: Optimal resource allocation policy (left) for a given value of $s_{1}=100$ and (right) for a given value of $d_{1}=60$

For $s_{1}=100$, the structure of the optimal allocation policy with respect to $d_{1}$ is illustrated in Figure 4 (left) for the two-node example in Figure 3. The threshold value is $T_{d}(100)=45$. If demand at node 1 is relatively small (i.e., $d_{1} \leq T_{d}(100)=45$ ), then demand at node 1 can be fully satisfied with sufficient supply left to satisfy demand at the second node. For $d_{1}>T_{d}(100)=45$, it is not optimal to fulfill node 1 's demand in full, $x_{1}^{*}=H_{1}\left(100, d_{1}\right)<d_{1}$, and as $d_{1}$ increases, supply must still be held for the second node. Therefore, the allocation will not increase at the same rate as demand, and $H_{1}\left(100, d_{1}\right)$ is an increasing concave function of $d_{1}$.

Theorem 2. (Sensitivity of the Optimal Resource Allocation Policy to Supply) In a twonode SRA-e problem, for a given demand $d_{1}$ there exists a supply level, $T_{s}\left(d_{1}\right)$, such that:

(i) for $s_{1}<T_{s}\left(d_{1}\right)$, the optimal allocation, $x_{1}^{*}=H_{1}\left(s_{1}, d_{1}\right)<d_{1}$, where $H_{1}\left(s_{1}, d_{1}\right)$ is an increasing linear function of $s_{1}$ (i.e., $\left.H_{1}\left(s_{1}, d_{1}\right)=\alpha s_{1}\right)$. Furthermore, the optimal objective function value at node $1, Z^{(1)}\left(s_{1}, 1, d_{1}\right)$ is increasing with supply $s_{1}$ at a constant ratio, $\theta$. 
(ii) for $s_{1} \geq T_{s}\left(d_{1}\right)$, the optimal allocation, $x_{1}^{*}=d_{1}$.

(iii) $T_{s}\left(d_{1}\right)$, $\alpha$ and $\theta$ are non-decreasing in $d_{1}$.

If $s_{1}=T_{s}\left(d_{1}\right)$, then $x_{1}^{*}=d_{1}$ and the optimal allocation does not increase with increased supply since allocations cannot exceed demand. However, for $s_{1}<T_{s}\left(d_{1}\right)$, then $x_{1}^{*}<d_{1}$; intuitively, the allocation will increase to raise the expected minimum fill rate. Further, Theorem 2 states that the optimal value, $Z^{(1)}\left(s_{1}, 1, d_{1}\right)$ increases linearly with $s_{1}$ if $s_{1}<T_{s}\left(d_{1}\right)$ at a rate which is independent of supply. This rate is solely determined by the observed demand at node 1 and the demand distribution at node 2 .

For $d_{1}=60$, Figure 4 (right) illustrates the allocation policy with respect to $s_{1}$ for the two-node example from Figure 3. In this figure, $T_{s}\left(d_{1}\right)=120$ and $\alpha=0.5$. Note that for $s_{1}=50, x_{1}^{*}=25<d_{1}$. In this case, as $s_{1}$ increases, $x_{1}^{*}$ increases linearly at a rate of $\alpha$ until $s_{1}=T_{s}\left(d_{1}\right)$ and $x_{1}^{*}=60$.

Since $x_{1}^{*}=T_{s}\left(d_{1}\right)$ for $d_{1}=T_{s}\left(d_{1}\right)$, the results from Theorem $2(i$ and $i i)$ imply that $T_{d}\left(s_{1}\right)$ is nondecreasing in $s_{1}$. Similarly, the results from Theorem $1(i$ and $i i)$ imply that $T_{s}\left(d_{1}\right)$ is a non-decreasing concave function of $d_{1}$; and since, $d_{1}=\alpha T_{s}\left(d_{1}\right)$, then $\alpha$ is also non-decreasing in $d_{1}$.

\section{Impact of Variability}

Using a simple case, we show counterintuitive behavior of the objective function with respect to variability of demand. Suppose that the demand distributions of both nodes of a two-node SRA-e follow discrete symmetric distributions with the same mean, shown in Figure 5(left), where the value of $p_{i}$ is different for each node.
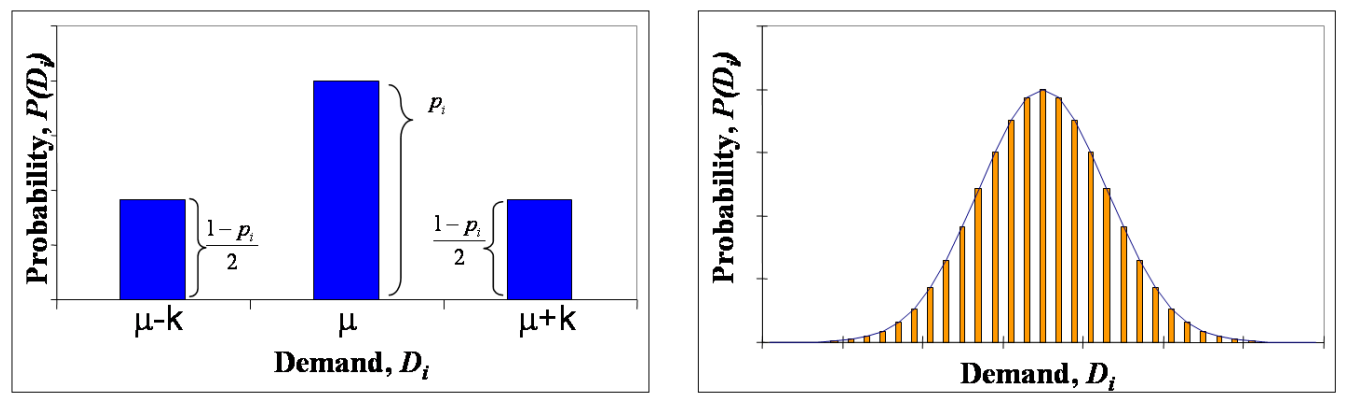

Figure 5: left: Discrete demand distribution; $k<\mu$, and $p_{i} \geq \frac{1-p_{i}}{2}$. right: Discretizing a continuous demand distribution

Proposition 1. Given two nodes following the demand distributions in Figure 5(left), there exists a supply $s^{\prime}$, such that if $s_{0} \leq s^{\prime}$, increasing the demand variability of node 1 (by decreasing $p_{1}$ ) increases 
the expected minimum fill rate, where

$$
s^{\prime}=\frac{2 \mu(2 \mu+k)\left(p_{2} \mu+k\right)}{\left(1+p_{2}\right)(\mu+k)^{2}-\left(1-p_{2}\right) \mu^{2}} .
$$

Proposition 1 claims that if $s_{0}$ is less than threshold $s^{\prime}$, then increasing the variability in demand in node 1 improves the objective function, which is contrary to common intuition that demand variability worsens system performance. In our SRA-e model, we observe several cases where increasing demand variability leads to higher system performance. One reason for these dynamics is that low supply levels result in low fill rates. In this case, increasing demand variability increases the probability of lower demand observations, and therefore increases the expected fill rates. For high supply levels, lower demand observations are satisfied fully resulting in a fill rate of 1 , which is the maximum; therefore, the solution does not benefit from the increased probability of lower demand values.

Note that by including more levels of demands, this discrete representation of a distribution in Proposition 1 can be generalized to mimic the shape of symmetric unimodal continuous probability distributions as shown in Figure 5(right). While this exponentially increases the number of combinations to consider in the analysis, it does not change the derived insight from Proposition 1.

Numerically solving for the optimal resource allocation policy for the SRA-e is computationally intensive since the optimality equation, $Z^{(i)}\left(s_{i}, \beta_{m i n}^{i-1}, d_{i}\right)$, is evaluated for a three-dimensional state space. To numerically evaluate $\mathcal{Z}\left(s_{0}\right)$, it is necessary to discretize the state space. Let $\ell_{s}, \ell_{\beta}$ and $\ell_{d}$ represent the number of discretization intervals for supply, fill-rate and demands, respectively. The number of intervals for the resource allocation $\left(\ell_{x_{i}}\right)$ is dependent on supply, demand and fill-rate. If $s_{i} \geq d_{i}$, then $\ell_{x_{i}}=\ell_{\beta} \beta_{\text {min }}^{i-1}$, since $x_{i}$ is constrained by the minimum fill rate and is chosen to meet the fill rate intervals. If $s_{i}<d_{i}$, then $x_{i}$ is also limited by supply, thus $\ell_{x_{i}}=\ell_{\beta}\left(\beta_{\min }^{i-1} \wedge \frac{s_{i}}{d_{i}}\right)$. For an $N$-node SRA-e, at node $N$, there are $\ell_{s} \ell_{\beta} \ell_{d}$ possible states, and $Z^{(N)}(\cdot)$ is determined with one computation for each state since the optimal allocation policy is $s_{N} \wedge d_{N}$. For nodes 2 through $N-1$ there are also $\ell_{s} \ell_{\beta} \ell_{d}$ possible states each, and $Z^{(i)}(\cdot)$ is evaluated with $\ell_{x_{i}}$ computations for each state to determine the optimal allocation. For node 1 , there are $\ell_{d}$ possible states since $s_{1}=s_{0}$ and $\beta_{\text {min }}^{0}=1$; the evaluation of $Z^{(0)}(\cdot)$ requires $\ell_{x_{1}}$ computations for each state to determine the optimal allocation. In total, the number of computations needed to evaluate $\mathcal{Z}\left(s_{0}\right)$ is equal to $\ell_{d} \ell_{x_{1}}+\sum_{i=1}^{N-1}\left(\ell_{s} \ell_{\beta} \ell_{d} \ell_{x_{i}}\right)+\ell_{s} \ell_{\beta} \ell_{d}$. For a three-node SRA-e, where supply and fill rate are discretized into 100 intervals and demand is discretized into 20 intervals, the number of computations necessary to determine $x_{1}^{*}$ and evaluate $\mathcal{Z}\left(s_{0}\right)$ 
is on the order of $10^{8}$. Further, the computations needed to solve the dynamic program grow with the number of nodes. This highlights the need for near-optimal heuristics that are easy to calculate. We consider heuristic policies which produce allocation policies that mimic the structure of the optimal policy and result in a near-optimal objective value.

\subsection{Resource Allocation Heuristics}

With the insights from two-node models, we develop a heuristic solution for the $N$-node problem which we call the Two-node Decomposition (TND) heuristic. TND decomposes the $N$-node SRA-e into sequential two-node problems; therefore, we first develop a two-node allocation heuristic.

\subsubsection{Two-Node Problem}

Based on Theorem 1, for a given supply at node 1 in a two-node problem, the optimal allocation policy can be reasonably approximated by a piecewise function consisting of a linear function and a concave function. Therefore, a two-node allocation heuristic policy at node $1, \hat{x}_{1}$, would take on the structure: $\hat{x}_{1}=\min \left\{\hat{H}_{1}\left(s_{1}, d_{1}\right), d_{1}\right\}$, where $\hat{H}_{1}\left(s_{1}, d_{1}\right)$ is an approximation of $H_{1}\left(s_{1}, d_{1}\right)$.

Suppose $\rho$ is a forecast for demand at node 2. Using this forecast, our heuristic obtains $\hat{H}_{1}\left(s_{1}, d_{1}\right)$ by solving $\frac{\hat{H}_{1}\left(s_{1}, d_{1}\right)}{d_{1}}=\frac{s_{1}-\hat{H}_{1}\left(s_{1}, d_{1}\right)}{\rho}$, which yields $\hat{H}_{1}\left(s_{1}, d_{1}\right)=s_{1} \frac{d_{1}}{d_{1}+\rho}$. We plot $\hat{x}_{1}$ as a function of $d_{1}$ in Figure 6 for $s_{1}=100$ and an arbitrary value for $\rho$; i.e., $\rho=55$.

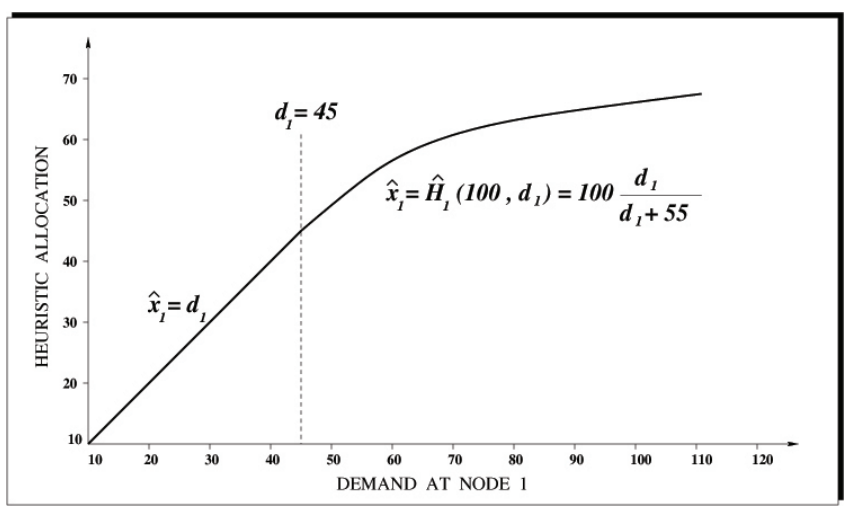

Figure 6: Heuristic allocation policy

The heuristic allocation curve in Figure 6 is similar in shape to the optimal allocation curve in Figure $4($ left). Note that if forecast $\rho$ is accurate (i.e., $\rho$ is exactly equal to the demand at node 2), then our heuristic results in the optimal allocation policy (i.e., $\left.\hat{H}_{1}\left(s_{1}, d_{1}\right)=H_{1}\left(s_{1}, d_{1}\right)\right)$. If one cannot guarantee a precise value for $\rho$, which is generally the case, one must choose $\rho$ such that $\hat{H}_{1}\left(s_{1}, d_{1}\right)$ approximates $H_{1}\left(s_{1}, d_{1}\right)$ as accurately as possible. 
It is clear that $\rho$ depends on the magnitude of and variability in the demand of node 2. Our heuristic bases the value of $\rho$ on the median of the demand $\left(\tilde{m}_{2}\right)$, to capture the magnitude, adjusted by a correction factor $\left(\delta_{2} \sqrt{\sigma_{2}}\right)$, to account for demand variability. Specifically, $\rho$ is calculated as

follows: $\rho=\tilde{m}_{2}+\delta_{2} \sqrt{\sigma_{2}}$, where $\delta_{2}=\frac{\tilde{m}_{1}-\tilde{m}_{2}}{\operatorname{avg}\left(\tilde{m}_{1}, \tilde{m}_{2}\right)}$, which is the difference between median demands standardized by the average median demand.

The correction factor $\left(\delta_{2} \sqrt{\sigma_{2}}\right)$, consists of $\sigma_{2}$ to account for demand variability in node 2 , and $\delta_{2}$ to account for dissimilarity in the demand magnitude between nodes 1 and 2. Dissimilarity in the demand magnitude affects fill rate value of supply at each node. For example, if demand at node 1 is significantly lower than that at node 2 , then increasing the allocation to node 1 by one unit has more impact on its fill rate than increasing the allocation to node 2 by one unit. In this case the correction factor would be negative, since $\tilde{m}_{1}<\tilde{m}_{2}$, to reduce the value of $\rho$. Similarly, if the demand magnitude at node 1 is significantly greater than that at node 2 , then the fill rate value of supply at node 2 is greater and the correction factor would increase the value of $\rho$.

From numerical studies, we find that basing $\rho$ on median is more effective than basing $\rho$ on mean for a wider variety of demand distributions. For example, if the demand at node 2 is strongly skewed to the left, the probability of demand being significantly more than $\mu$ is much larger than 0.5. Basing $\rho$ on mean would assign too small a weight on node 2's demand and therefore, allocate too much to node 1. However, when $\rho$ is based on median, regardless of the shape of the distribution, the probability that the demand be smaller or larger than median is always 0.5 , so the inventory reserved for node 2 will not be too large or too small. A similar argument holds for demand distributions that are skewed to the right. For symmetric distributions, using median is identical to using the mean. The complete two-node allocation heuristic can be written:

$$
\begin{gathered}
\hat{x}_{1}=\min \left\{\hat{H}_{1}\left(s_{1}, d_{1}\right), d_{1}\right\} \\
\hat{H}_{1}\left(s_{1}, d_{1}\right)=s_{1} \frac{d_{1}}{d_{1}+\left(\tilde{m}_{2}+\delta_{2} \sqrt{\sigma_{2}}\right)}
\end{gathered}
$$

\subsubsection{N-Node Problem}

The TND heuristic consists of three phases:

Phase 1. Decomposition: The $N$-node problem is decomposed into sequential two-node problems. For example, at node 1, the TND heuristic solves a two-node SRA-e consisting of nodes 1 and 2. At node 2, the method solves another two-node SRA-e consisting of nodes 2 and 3, and so on. 
Phase 2. Supply Allotment: For each sequential two-node SRA-e, the available supply is divided so that one portion is allotted to solve the two-node problem and the other is reserved for the other nodes not yet visited. For example, for $N=5$, a portion of $s_{1}$ is allotted for the two-node problem for nodes 1 and 2, and the rest is reserved for nodes 3, 4 and 5. At node 2, a portion of $s_{2}$ is alloted for the two-node problem for nodes 2 and 3 , and the rest is reserved for remaining nodes 4 and 5 , and so on. The allotment $\left(\hat{s}_{i}\right)$ for the two-node SRA-e for nodes $i$ and $i+1$ is determined by:

$$
\hat{s}_{i}=s_{i} \frac{\mu_{i}+\mu_{i+1}}{\sum_{j=i}^{N} \mu_{j}}
$$

At each visit, $\hat{s}_{i}$ is calculated so that an appropriate amount of supply is available for allocation to the two-node problem $i$ and $i+1$. The fraction of available supply $s_{i}$ that is allotted to the two-node problem, $\hat{s}_{i}$, is proportional to the average demand of the two node relative to the total average demand of the unvisited nodes. Note that, although one can also base the allotment on median, we observed in our numerical study that using medians instead of means to determine supply allotments reduces the effectiveness of the TND algorithm.

Phase 3. Resource Allocation: For each sequential two-node problem consisting of nodes $i$ and $j$ with the alloted supply $\hat{s}_{i}$ determined by (10), the two-node allocation heuristic is used to determine the allocation amount:

$$
\begin{gathered}
\hat{x}_{i}=\min \left\{\hat{H}_{i}\left(\hat{s}_{i}, d_{i}\right), \beta_{\text {min }}^{i-1} d_{i}\right\} \\
\hat{H}_{i}\left(\hat{s}_{i}, d_{i}\right)=\hat{s}_{i} \frac{d_{i}}{d_{i}+\left(\tilde{m}_{i+1}+\delta_{i+1} \sqrt{\sigma_{i+1}}\right)}
\end{gathered}
$$

Note that (11) includes the term $\beta_{m i n}^{i-1}$, the minimum fill rate among nodes visited, which differs from (8). In the $N$-node problem, $\beta_{\text {min }}^{i-1}$ depends on the observed demand and allocation decisions at nodes 1 to $i-1$; in the two-node problem, $\beta_{\text {min }}^{0}=1$. Allocation according to (11) implies that there is no incentive to allocate an amount that will result in a higher fill rate than $\beta_{\text {min }}^{i-1}$, which is the current minimum fill rate since the objective is to maximize the minimum fill rate of the entire sequence. One would rather reserve supply to address future demand volatility.

The TND heuristic algorithm begins at node 1 with initial supply, $s_{0}$, and steps through the three phases as each node is visited. The following is a complete description of the algorithm: 


\section{TND Heuristic Allocation Algorithm}

Step 0: $\quad$ Initialize $i=1, s_{1}=s_{0}$ and $\beta_{\text {min }}^{0}=1$.

Step 1: Determine supply allotment, $\hat{s}_{i}$, using $\hat{s}_{i}=s_{i} \frac{\mu_{i}+\mu_{i+1}}{\sum_{j=i \ldots N} \mu_{j}}$.

Step 2: Determine resource allocation, $\hat{x}_{i}$, for the two-node problem for nodes $i$ and $i+1$ after observing $d_{i}$ : using $\hat{x}_{i}=\min \left\{\hat{H}_{i}\left(\hat{s}_{i}, d_{i}\right), \beta_{\min }^{i-1} d_{i}\right\}$, where $\hat{H}_{i}\left(\hat{s}_{i}, d_{i}\right)=\hat{s}_{i} \frac{d_{i}}{d_{i}+\left(\tilde{m}_{i+1}+\delta_{i+1} \sqrt{\sigma_{i+1}}\right)}$.

Step 3: Update $\beta_{\text {min }}^{i}=\beta_{\text {min }}^{i-1} \wedge \frac{\hat{x}_{i}}{d_{i}}$ and $s_{i+1}=s_{i}-\hat{x}_{i}$.

Step 4: If $i+1<N$, then set $i=i+1$ and go to Step 1. Else $i+1=N$ STOP; $\hat{x}_{N}=s_{N} \wedge d_{N}$.

Note that the TND heuristic algorithm reduces to the two-node allocation heuristic when $N=2$.

\subsubsection{Excess Priority and Excess Sharing Heuristics}

We evaluate the performance of the TND heuristic against the optimal allocation policy, as well as four heuristic allocation policies developed as benchmarks to evaluate the performance of our TND heuristic: (i) Excess Priority heuristic based on mean, (ii) Excess Priority heuristic based on median, (iii) Excess Sharing heuristic based on mean, and (iv) Excess Sharing heuristic based on median. All of these heuristics consider a threshold value $\kappa_{i}$ for node $i$, and allocate inventory $x_{i}$ to node $i$ as follows:

$$
x_{i}=\left\{\begin{array}{lll}
d_{i} & : & \text { if } d_{i} \leq \kappa_{i} \\
\kappa_{i} & : & \text { if } d_{i}>\kappa_{i}
\end{array}\right.
$$

\section{Excess Priority Heuristic Based on Mean:}

Under this heuristics, initial values for thresholds $\kappa_{i}^{(o)}$, for all $i$, are calculated based on the mean of demand at all nodes as follows:

$$
\kappa_{i}^{(o)}=s_{0} \frac{\mu_{i}}{\sum_{j=1}^{N} \mu_{j}} .
$$

These initial thresholds are updated after the demand at a node is observed. Specifically, after visiting node $i$, if there is excess supply (i.e., if node $i$ requests less than its assigned threshold), then the excess is available immediately for allocation to node $i+1$. Under Excess Priority heuristics, after allocation at node $i$, only the threshold value at node $i+1$ is updated. More formally, suppose that $\kappa_{i+1}^{(i)}$ is the updated threshold at node $i+1$ after nodes 1 to $i$ are visited. Then, after visiting node $i$, the value of $\kappa_{i+1}^{(i)}$ for node $i+1$ is obtained as follows:

$$
\kappa_{i+1}^{(i)}=\left\{\begin{array}{lll}
\kappa_{i+1}^{(o)}+\left(\kappa_{i}^{(i-1)}-d_{i}\right) & : & \text { if } d_{i} \leq \kappa_{i}^{(i-1)} \\
\kappa_{i+1}^{(o)} & : & \text { if } d_{i}>\kappa_{i}^{(i-1)}
\end{array}\right.
$$

Note that, upon arrival to node $i+1$, the heuristic tends to allocate all the excess (unused) supply at node $i$ (i.e., $\kappa_{i}^{(i-1)}-d_{i}$ ) to node $i+1$ by increasing the initial threshold at node $i+1$ from $\kappa_{i+1}^{(o)}$ to 
$\kappa_{i+1}^{(o)}+\left(\kappa_{i}^{(i-1)}-d_{i}\right)$. If there is no excess supply at node $i$, then the initial allocation threshold at node $i+1$ does not change.

The Excess Priority Heuristic Based on Median is analogous to the above, except median $\tilde{m}_{i}$ is used in place of mean $\mu_{i}$ for all $i=1,2, \ldots, N$.

\section{Excess Sharing Heuristic Based on Mean:}

The Excess Sharing Heuristics use the same initial values for threshold as in (13). However, after visiting node $i$, if there is excess supply (i.e., if the demand in node $i$ is less than its assigned threshold), then the excess is shared among all remaining nodes, by revising all thresholds in those nodes.

Specifically, after visiting node $i$, the thresholds $\kappa_{j}^{(i)}$ are revised for all nodes $j=i+1, i+2, \ldots, N$ as follows:

$$
\kappa_{j}^{(i)}=\left\{\begin{array}{lll}
\kappa_{j}^{(i-1)}+\left(\kappa_{i}^{(i-1)}-d_{i}\right) \frac{\mu_{j}}{\sum_{k=i+1}^{N} \mu_{k}} & : & \text { if } d_{i} \leq \kappa_{i}^{(i-1)} \\
\kappa_{j}^{(i-1)} & : & \text { if } d_{i}>\kappa_{i}^{(i-1)}
\end{array}\right.
$$

The Excess Sharing Heuristic Based on Median is analogous to the above, except median $\tilde{m}_{i}$ is used in place of mean $\mu_{i}$ for all $i=1,2, \ldots, N$.

\subsubsection{Performance Evaluation of Allocation Heuristics}

In the numerical study, we evaluate the performance of our TND, excess priority, and excess sharing heuristics with that of the optimal allocation policy

In our numerical studies, we consider instances with 2 to 7 nodes. In the FRP, the majority of routes visit 7 agencies or less. Demands are observed from gamma distributions, which allow the modeling of settings with low and high variability corresponding to coefficient of variation (CV) less and greater than 1. Demand distributions are discretized into equally spaced intervals and all possible sample paths are enumerated to calculate the performance of each allocation policy. Table 1 summarizes the SRA-e scenarios in our analysis, which can be organized into three categories.

Node sets A to D consist of nodes with identical demand means, either low (50) or high (150). The CVs of the demand within the sets are uniformly distributed over a range. We consider a wide range of $\mathrm{CV}(0.5-1.5)$ and a narrow range $(0.75-1.25)$. For example, for an instance with 5 nodes with the wide range of $\mathrm{CV}$, the CV's assigned to the 5 nodes are $0.5,0.75,1.0,1.25$, and 1.5. For node sets $\mathrm{A}$ to $\mathrm{D}$, we consider five sequences: The $C V^{\nearrow}$ sequence visits nodes in order of lowest to highest $\mathrm{CV}$, and the $C V^{\searrow}$ sequence visits nodes in order of highest to lowest $\mathrm{CV}$. The $C V^{\wedge}$ sequence places the higher CV nodes in the middle of the route and the lower CV nodes at the beginning and 


\begin{tabular}{|c|c|c|l|}
\hline Node set & $\mu$ & CV & Sequences \\
\hline \hline $\mathrm{A}$ & 50 & $0.5-1.5$ & \\
$\mathrm{~B}$ & 150 & $0.5-1.5$ & $C V^{\nearrow}, C V^{\searrow}, C V^{\wedge}, C V^{\vee}, C V^{\sim}$ \\
$\mathrm{C}$ & 50 & $0.75-1.25$ & \\
$\mathrm{D}$ & 150 & $0.75-1.25$ & \\
$\mathrm{E}$ & $50-150$ & 0.5 & $\mu^{\nearrow}, \mu \searrow, \mu^{\wedge}, \mu^{\vee}, \mu^{\sim}$ \\
$\mathrm{F}$ & $50-150$ & 1.5 & \\
$\mathrm{G}$ & $75-125$ & 0.5 & \\
$\mathrm{H}$ & $75-125$ & 1.5 & \\
\hline $\mathrm{I}$ & $50-150$ & Large $\mu$ with large CV & $C V^{\nearrow}, C V^{\searrow}, C V^{\wedge}, C V^{\vee}, C V^{\sim}$ \\
& & $0.5-1.5$ & \\
$\mathrm{~J}$ & $50-150$ & Large $\mu$ with small CV & \\
\hline
\end{tabular}

Table 1: SRA-e scenarios in numerical study

end of the routes. The $C V^{\vee}$ sequence is the opposite, where the higher CV nodes are at the beginning and end of the routes, and lower CV nodes are in the middle of the route. Finally, the $C V^{\sim}$ sequence alternates the nodes with low and high CV.

Node sets $\mathrm{E}$ to $\mathrm{H}$ consist of nodes with identical CV, either low (0.5) or high (1.5). The mean demand within the node sets are uniformly distributed over a wide range (50 - 150) or narrow range (75 - 125). We consider five sequences: $\mu^{\nearrow}, \mu^{\searrow}, \mu^{\wedge}, \mu^{\vee}$ and $\mu^{\sim}$ which follow the same sequencing logic as CV sequencing.

Node sets I and J, consist of nodes with different $\mu$ and $\mathrm{CV} ; \mu$ is uniformly distributed from 50 to 150 and $\mathrm{CV}$ is distributed from 0.5 to 1.5 . In node set $\mathrm{I}$, the nodes with large $\mu$ have high $\mathrm{CV}$ and in node set $\mathrm{J}$, the nodes with large $\mu$ have low CV. For both sets, we consider the same sequences as node sets A to D.

We consider the five sequences for each of the ten node sets in Table 1, resulting in 50 different routes for instances with 3 to 7 nodes. In two-node instances there are only two possible sequences resulting in 20 different routes. For each of these 270 routes, we consider five levels of initial supply ranging from 0.5 to 1.5 times the total mean demand of the nodes in a route (i.e., $\sum_{i=1}^{N} \mu_{i}$ ). In total, our study evaluates each allocation policy in 1,350 different scenarios.

Let $g_{H}$ represent the objective value for the TND heuristic, and let $g_{\mu}$ and $g_{\tilde{m}}$ represent the objective values for the corresponding mean and median allocation heuristic policies, respectively. Table 2 lists the average and maximum optimality gaps (i.e. $\mathcal{Z}-g_{h}$ ) for the five policies considered. In our numerical analysis we observe the following: 


\begin{tabular}{|c|c|c|c|c|c|c|c|c|c|c|}
\hline \multirow{3}{*}{$\begin{array}{c}\text { Number of } \\
\text { nodes }\end{array}$} & \multirow{2}{*}{\multicolumn{2}{|c|}{$\frac{\text { TND }}{\mathcal{Z}-g_{H}}$}} & \multicolumn{4}{|c|}{ Excess Priority } & \multicolumn{4}{|c|}{ Excess Sharing } \\
\hline & & & \multicolumn{2}{|c|}{$\mathcal{Z}-g_{\mu}$} & \multicolumn{2}{|c|}{$\mathcal{Z}-g_{\tilde{m}}$} & \multicolumn{2}{|c|}{$\mathcal{Z}-g_{\mu}$} & \multicolumn{2}{|c|}{$\mathcal{Z}-g_{\tilde{m}}$} \\
\hline & Avg & Max & Avg & $\operatorname{Max}$ & Avg & Max & Avg & $\operatorname{Max}$ & Avg & Max \\
\hline 2 & $0.6 \%$ & $2.6 \%$ & $3.0 \%$ & $15.5 \%$ & $4.9 \%$ & $23.9 \%$ & $3.0 \%$ & $15.5 \%$ & $4.9 \%$ & $23.9 \%$ \\
\hline 3 & $0.7 \%$ & $3.0 \%$ & $4.7 \%$ & $12.7 \%$ & $6.8 \%$ & $26.9 \%$ & $5.4 \%$ & $13.2 \%$ & $8.4 \%$ & $27.7 \%$ \\
\hline 4 & $0.8 \%$ & $2.9 \%$ & $6.2 \%$ & $13.5 \%$ & $7.7 \%$ & $29.8 \%$ & $8.1 \%$ & $14.7 \%$ & $10.3 \%$ & $31.9 \%$ \\
\hline 5 & $1.2 \%$ & $3.2 \%$ & $7.6 \%$ & $14.0 \%$ & $9.1 \%$ & $33.4 \%$ & $10.5 \%$ & $18.0 \%$ & $12.9 \%$ & $37 \%$ \\
\hline 6 & $1.7 \%$ & $4.2 \%$ & $8.8 \%$ & $14.5 \%$ & $10.4 \%$ & $36.1 \%$ & $12.8 \%$ & $21.0 \%$ & $15.2 \%$ & $40.8 \%$ \\
\hline 7 & $2.3 \%$ & $4.9 \%$ & $9.8 \%$ & $15.5 \%$ & $11.5 \%$ & $37.2 \%$ & $14.8 \%$ & $23.7 \%$ & $17.2 \%$ & $43.0 \%$ \\
\hline Total & $1.3 \%$ & $4.9 \%$ & $7.1 \%$ & $15.5 \%$ & $8.8 \%$ & $37.2 \%$ & $9.1 \%$ & $23.7 \%$ & $11.5 \%$ & $43.0 \%$ \\
\hline
\end{tabular}

Table 2: Average and maximum optimality gaps

- For all policies, the average optimality gaps increase with the number of nodes. On average, the TND heuristic is within $1.3 \%$ of the optimal allocation policy and at worst less than $5 \%$ from optimal for routes up to seven nodes. The TND heuristic errors trend upward for larger problems; however, the heuristic's performance is significantly better than that of the other four heuristics in most cases.

- In a direct comparison, the TND heuristic is, on average, $5.8 \%$ and $7.5 \%$ better than the excess priority mean and median objectives, respectively, with maximum differences of $15.4 \%$ and $32.3 \%$. Even though the differences between the TND heuristic and excess priority median heuristic is large, there are cases in which the excess priority median heuristic results in a higher objective than the TND heuristic. These cases are typically in node sets A, B, I and J with the $C V^{\nearrow}$ sequence. The differences are, on average, less than $0.5 \%$ and as high as $1.5 \%$, which are relatively small when compared to the difference in cases where the TND heuristic is better. For node sets $\mathrm{A}, \mathrm{B}, \mathrm{I}$ and $\mathrm{J}$, where nodes are visited in $C V^{\nearrow}$ sequence, the optimality gap of the excess priority median heuristic is much smaller than the aggregated results in Table 2. For these scenarios, the average gaps range from $0.7 \%$ for a two-node problem to $3.7 \%$ for a seven-node problem. Therefore, in these cases, both the excess priority median and the TND heuristic are effective approximations for the optimal policy.

- The excess priority mean and median heuristics are, on average, $2.0 \%$ and $2.7 \%$ better than the excess sharing mean and median heuristics, respectively. This may seem counterintuitive since the excess sharing policy seems more equitable as it redistributes the excess supply among all nodes not yet visited. However, this redistribution is calculated based on the assigned thresholds that may not be optimal. Therefore, it is better to use the excess supply as needed now (when the 
demand is realized) rather than redistributing the excess among remaining nodes, expecting that they will definitely be needed later in the remaining nodes. This, as we observed in our numerical study, leads to excess priority heuristic producing less waste than excess sharing heuristic, and therefore a more effective inventory allocation.

In Table 3, we present the average and maximum optimality gaps, as well as the percent of cases in which the TND objective is within $2 \%$ of the optimal value for each node set. The detail results for Excess Priority and Excess Sharing heuristics are presented in On-Line Appendix A. The TND heuristic performs within $2 \%$ of the optimal allocation policy for a significant majority of cases. For scenarios with higher demand variability ( sharpest comparison is the difference between sets $\mathrm{E}$ and $\mathrm{G}$ to $\mathrm{F}$ and $\mathrm{H}$, respectively, which are sets with the same mean but with different CV. The TND heuristic is based on approximating demand, and therefore will have lower optimality gaps for settings with more predictable demand such as in sets $\mathrm{E}$ and $\mathrm{G}$. However, for settings with more demand variability as in sets $\mathrm{F}$ and $\mathrm{H}$, the average optimality gap is less than $2 \%$ and in $77 \%$ of the cases in these two sets, the performance of the heuristic is within $2 \%$ of that of the optimal allocation policy.

\begin{tabular}{|c|cccccccccc|}
\hline & A & B & C & D & E & F & G & H & I & J \\
\hline Average optimality gap & $1.1 \%$ & $1.2 \%$ & $1.1 \%$ & $1.1 \%$ & $0.8 \%$ & $1.8 \%$ & $0.7 \%$ & $1.8 \%$ & $1.4 \%$ & $1.2 \%$ \\
Maximum optimality gap & $3.6 \%$ & $3.7 \%$ & $3.1 \%$ & $3.0 \%$ & $3.2 \%$ & $4.9 \%$ & $2.6 \%$ & $4.2 \%$ & $4.3 \%$ & $4.4 \%$ \\
\% of cases gap $\leq 2 \%$ & $93 \%$ & $88 \%$ & $91 \%$ & $91 \%$ & $97 \%$ & $83 \%$ & $99 \%$ & $71 \%$ & $87 \%$ & $90 \%$ \\
\hline
\end{tabular}

Table 3: TND heuristic optimality gaps by node sets

The numerical studies show that the TND heuristic determines an allocation policy which results in significantly better objective values than the benchmark policies. The heuristic's objective is within $2 \%$ of an optimal allocation policy in about $90 \%$ of the cases. Although the gaps of the TND heuristic increase with route size, they remain significantly better than the benchmark heuristics errors which increase at a higher rate. Given the service time at a node and the travel time between nodes, many pick-up and delivery applications are limited in the number of stops per route, see pickup and delivery test cases presented in Savelsbergh and Sol (1995). Service times in the FRP are approximately 10-20 minutes for donors and 15-30 minutes for agencies, and travel distances between nodes are between 10 to 20 minutes. It is easy to see that routes are not likely to visit more than 7 agencies, since donors must be visited on routes as well. Our heuristic is valuable as well in applications with many nodes per route, since the computational complexity does not allow one to compute the optimal allocation 
policy.

\section{$5 \quad$ Sequencing Policies}

Sequencing nodes in the SRA-e along a route impacts the expected minimum fill rate, as shown in Section 4. In the case of the FRP, this can also impact the distance traveled and the associated travel cost. However, if travel costs are low compared to stopping costs, or if all locations are located in a dense area (such as the agencies in Figure 1), then the total distances or costs of the visitation sequences do not differ significantly. In such settings, it may be beneficial to find the best sequence of nodes that optimizes the fill rate objective.

The impact of sequencing can be significant; consider the example in Figure 2. Let the demand at node 1 remain the same, but the demand at node 2 be either 10 or 90 with equal probability. When node 1 is visited first, given $s_{0}=130$, we have $\mathcal{Z}=70 \%$. If node 2 is visited first, then $\mathcal{Z}=82 \%$ which is $12 \%$ higher. This is a simple example that shows sequencing can have a significant impact on the objective value. In this section, we address the sequencing decision when given a set of nodes to visit. We begin with a study of optimal sequencing policies for special cases in Section 5.1. Based on these results we construct a sequencing heuristic for general problems, which we present in Section 5.2 .

\subsection{Optimal Sequencing Policy}

To investigate the impact of mean on the optimal sequence, we first study a two-node problem where nodes observe demands from the same family of distributions with different means, but with identical standard deviations. In this case, we are able to show that it is optimal to sequence the node with lower demand mean first.

Theorem 3. In a two-node SRA-e problem in which two nodes observe demand from the same family of distributions with different means, but with identical standard deviations, visiting the nodes in increasing order of demand mean will result in a higher expected minimum fill rate.

We now consider a case where nodes observe demand from distributions with identical means but different standard deviations, such as the discrete demand distribution in Figure 5(left) in Section 4.1. 
Theorem 4. In a two-node SRA-e problem in which two nodes follow the demand distributions in Figure 5(left), if $p_{1}<p_{2}$, then the expected minimum fill rate is greater when node 1 (which has a higher standard deviation) is visited first.

Theorem 4 suggests that it is optimal to sequence the nodes in decreasing order of standard deviation. This result is not surprising; it is intuitive that visiting the node with higher demand variability first is preferable. This sequencing minimizes the effect of variability, since the demand at the first node is observed and the uncertainty arises from the second node which has more predictable demand.

In Theorem 3, the demand variability of both nodes are identical in an absolute sense, since they have identical standard deviation. However, their coefficient of variations $(\mathrm{CV})$, a measure of relative demand variability, differ since the distributions have different means. The node with the smaller mean has higher $\mathrm{CV}$, and thus higher relative demand variability. The result in Theorem 3 implies that it is better to visit the node with higher relative demand variability first, which is consistent with the insight from Theorem 4.

Both Theorem 3 and 4 imply that relative $\mathrm{CV}$ can be a good rule for sequencing nodes. This principle of sequencing in decreasing order of CV is extended to more general problems next.

\subsection{Sequencing Heuristic}

Following Theorems 3 and 4, we define the following routing heuristic for more general problems.

Sequencing Heuristic: Sequence nodes in decreasing order of $C V$. If $C V$ 's are identical, sequence nodes in decreasing order of standard deviation.

We test the sequencing heuristic in two- to six-node instances with node sets described in Table 1 under the optimal resource allocation policy. For a $N$-node problem, the optimal sequence is found by comparing the objective function of all $N$ ! sequences under their corresponding optimal allocation policies. Therefore, we limit the tests to routes of six nodes or less. We consider five supply levels for the 10 node sets, resulting in 50 cases for each route size. In total, we evaluate the sequencing heuristic in 250 different scenarios. The results are summarized in Table 4.

The first row in Table 4 presents the percent of cases in which the heuristic determines the optimal sequence. The second row presents the percent of cases in which the difference between expected minimum fill rate of the heuristic sequence and that of the optimal sequence is less than $1 \%$. Lastly, 


\begin{tabular}{|c|ccccc|c|}
\hline & \multicolumn{5}{|c|}{ Number of nodes } & \multirow{2}{*}{ Total } \\
\cline { 2 - 6 } & 2 & 3 & 4 & 5 & 6 & $47 \%$ \\
\hline Optimal sequence chosen & $64 \%$ & $50 \%$ & $44 \%$ & $44 \%$ & $32 \%$ & $47 \%$ \\
Within 1\% of optimal sequence & $96 \%$ & $96 \%$ & $98 \%$ & $100 \%$ & $100 \%$ & $98 \%$ \\
Maximum optimality gap & $1.5 \%$ & $1.3 \%$ & $1.1 \%$ & $0.9 \%$ & $0.8 \%$ & $1.5 \%$ \\
\hline
\end{tabular}

Table 4: Effectiveness of the sequencing heuristic

the third row presents the maximum difference in objective function value between the sequencing heuristic and optimal sequence.

In general, in $47 \%$ of cases, the sequencing heuristic chooses the optimal sequence. These cases are typically in routes where nodes vary in CV; i.e., A, B, C, D, I and J. In these sets, the sequencing heuristic chooses the optimal sequence in $75 \%$ of cases. For node sets where nodes have identical CV (sets E, F, G and H), the sequencing heuristic chooses the optimal sequence in only $4 \%$ of these cases. However, the heuristic chooses a sequence within $1 \%$ of the optimal $98 \%$ of the time and the maximum difference in objective for all cases is $1.5 \%$. These results confirm that the sequencing heuristic is an effective method to determine a near-optimal visitation sequence.

\section{Resource Allocation Heuristic with Sequencing Heuristic}

In this section, we evaluate the performance of our sequencing and TND heuristics when used together to solve the SRA-e problem. For each node set described in Table 1, we determine the optimal sequence under optimal allocation and calculate the optimality gap in expected minimum fill rate from: the same optimal sequence under our TND heuristic $\left(S e q_{\text {opt }}\right.$ Alloc $\left.c_{\text {eu }}\right)$, our heuristic sequence under optimal allocation $\left(S e q_{h e u} A l l o c_{o p t}\right)$, and our heuristic sequence under our allocation heuristic $\left(S e q_{h e u} A l l o c_{h e u}\right)$. The results for two- to six-node problems, averaged over supply levels for each node set, are shown in Table 5.

\begin{tabular}{|c||cc|cc|cc|}
\hline \multirow{2}{*}{$\begin{array}{c}\text { Number of } \\
\text { nodes }\end{array}$} & \multicolumn{2}{|c|}{ Seq } & Alloc $_{\text {heu }}$ & \multicolumn{2}{|c|}{ Seq $_{\text {heu }}$ Alloc } \\
\cline { 2 - 7 } & Avg & Max & Avg & Max & Avg & Alloc \\
heu & Max \\
\hline \hline 2 & $0.5 \%$ & $2.6 \%$ & $0.2 \%$ & $1.5 \%$ & $1.0 \%$ & $2.6 \%$ \\
3 & $0.6 \%$ & $1.8 \%$ & $0.2 \%$ & $1.3 \%$ & $0.8 \%$ & $2.0 \%$ \\
4 & $1.0 \%$ & $2.6 \%$ & $0.2 \%$ & $1.1 \%$ & $0.9 \%$ & $1.7 \%$ \\
5 & $1.5 \%$ & $3.0 \%$ & $0.2 \%$ & $0.9 \%$ & $1.3 \%$ & $2.1 \%$ \\
6 & $2.1 \%$ & $4.2 \%$ & $0.2 \%$ & $0.8 \%$ & $1.8 \%$ & $3.3 \%$ \\
\hline Total & $1.1 \%$ & $4.2 \%$ & $0.2 \%$ & $1.5 \%$ & $1.2 \%$ & $3.3 \%$ \\
\hline
\end{tabular}

Table 5: Average and maximum optimality gaps

The results for $S e q_{o p t} A l l o c_{\text {heu }}$ consider only one sequence (i.e., the optimal sequence), and thus 
differ from the results in Table 2 that are averages over five sequences. The optimality gap values for $S e q_{\text {opt }}$ Alloc $_{\text {heu }}$ are consistent with those from Table 2, with low average and maximum values at $1.1 \%$ and $4.2 \%$, respectively. The results for $S e q_{h e u} A l l o c_{o p t}$ are from the same data summarized in Table 4 ; here, we include the averages. The average and maximum optimality gaps for $S_{e q_{h e u}} A l l_{o c} c_{h e u}$ are $1.2 \%$ and $3.3 \%$, respectively. These values are not significantly different from the gap values when only one heuristic is implemented, thus there is no compounding effect in combining the heuristics. We can conclude that combining the sequencing and TND heuristics results in near optimal solutions.

\section{$7 \quad$ Value of Information}

In some applications, it may be possible to obtain demand information prior to visiting the first node. In this section, we evaluate the benefit of advanced demand information. In Section 7.1, we compare the performance of the SRA-e in the case of unknown demands with the case where all demands are known (complete information). In Section 7.2, we consider a setting where demand information is only available from a single node (partial information).

\subsection{Complete Information}

Let $v_{c}$ represent the value of complete information: the absolute difference between the objective value when the demand at all nodes are known before visiting the first node and when demand is revealed only upon arrival at a node. We limit the numerical study to routes of six nodes and compute the maximum expected minimum fill rates for the routes described in Table 1, for a total of 250 scenarios. From our results, the average and maximum value of $v_{c}$ over all scenarios is $9.1 \%$ and $18.7 \%$, respectively. The average value of complete information is between $9 \%$ and $10 \%$ for each node set except $\mathrm{E}$ and $\mathrm{G}$, where the average $v_{c}$ is $6.8 \%$, due to the low $\mathrm{CV}$ values in these sets. Based on the data, we make the following observations:

Observation 1. The value of complete information $\left(v_{c}\right)$ is increasing with increasing variability of demand (i.e., $C V$ ) at all nodes. Furthermore, the value of information is decreasing as the initial supply $\left(s_{0}\right)$ becomes very large or very small.

Observation 2. For a given set of nodes, the maximum value of complete information $\left(v_{c}\right)$ is obtained when the nodes are sequenced in increasing order of demand variability.

As an example, consider scenarios in node set E and G with supply greater than the average demand 
of the route. The average value of complete information is only $1.2 \%$ and at most $2.5 \%$. In such scenarios, obtaining complete information does not provide significant benefit. In contrast, consider scenarios in node sets A, B, C, D, F, H, I and J with supply lower than the average demand of the route, and routed in either $C V^{\nearrow}$ or $\mu^{\nearrow}$. The average value of $v_{c}$ is $16.6 \%$ and at most $18.7 \%$. In these cases, obtaining complete information significantly improves the objective of maximizing the expected minimum fill rate.

The intuition behind Observation 1 is as follows. It is clear that the value of information increases in variability in demand, since as the variability in demand increases, predicting demand in nodes becomes more difficult. To understand the impact of initial supply on the value of information, consider an extreme case that the initial supply is very limited, e.g., $s_{0}=2$ and the average demand in each node of a three-node problem is $\mu_{i}=100$ for $i=1,2,3$. Note that, in this case, having complete demand information does not have much impact on the allocation decision, since the minimum expected fill rate would be zero, regardless of whether the demand information is utilized or not. However, as the initial supply increases, the demand information becomes more useful, and therefore the value of information increases. The value of information starts to decreases as the initial supply becomes very large. Consider the above example, and assume that the initial supply is $s_{0}=10,000$ instead of two. It is clear that the value of information is almost zero. Since ample supply is available for all nodes, the optimal decision is to allocate to each node whatever it requests, regardless of the demand information of remaining nodes. Therefore, the value of information decreases as initial supply becomes very large or very small.

Observation 2 states that the value of complete information is highest when nodes are sequenced in increasing order of demand variability. Note that when making an allocation decision at node $i$, the decision maker faces the uncertainty in demands in nodes $i+1$ to $N$. When nodes are sequenced in increasing order of demand variability, the decision making at each node $i(i=1,2, \ldots, N)$ involves more uncertainty compared to that of any other sequencing of nodes. Higher uncertainty corresponds to a higher value of information. Thus the value of information is highest in cases where nodes are sequenced in increasing order of demand variability.

\subsection{Partial Information}

In practice, it may be difficult to obtain demand information from all nodes. For example, in the FRP, obtaining demand information is difficult due to lack of personnel and the timing of delivery 
operations. It is more likely that demand information can be obtained from one or two nodes. We consider strategies of obtaining demand information from only one node and evaluate the portion of $v_{c}$ which is captured by observing demand from a single node. We address the following questions for the same six-node routes used in the numerical study for complete information:

1. Which position along the route provides the most valuable demand information? This excludes the first node, since its demand is known immediately before any decision is made.

2. Do the demand characteristics such as average demand $(\mu)$ and coefficient of variation (CV) of a node have an impact on the value of its demand information?

In answering question 1, for each node position along the route, we calculate the maximum expected minimum fill rate if demand from that node is obtained. Figure 7 presents the average objective values, from scenarios with node sets A-E, where information is obtained from the $i$ th node in the route, from no nodes and from all nodes. The results for node sets F-J are similar.

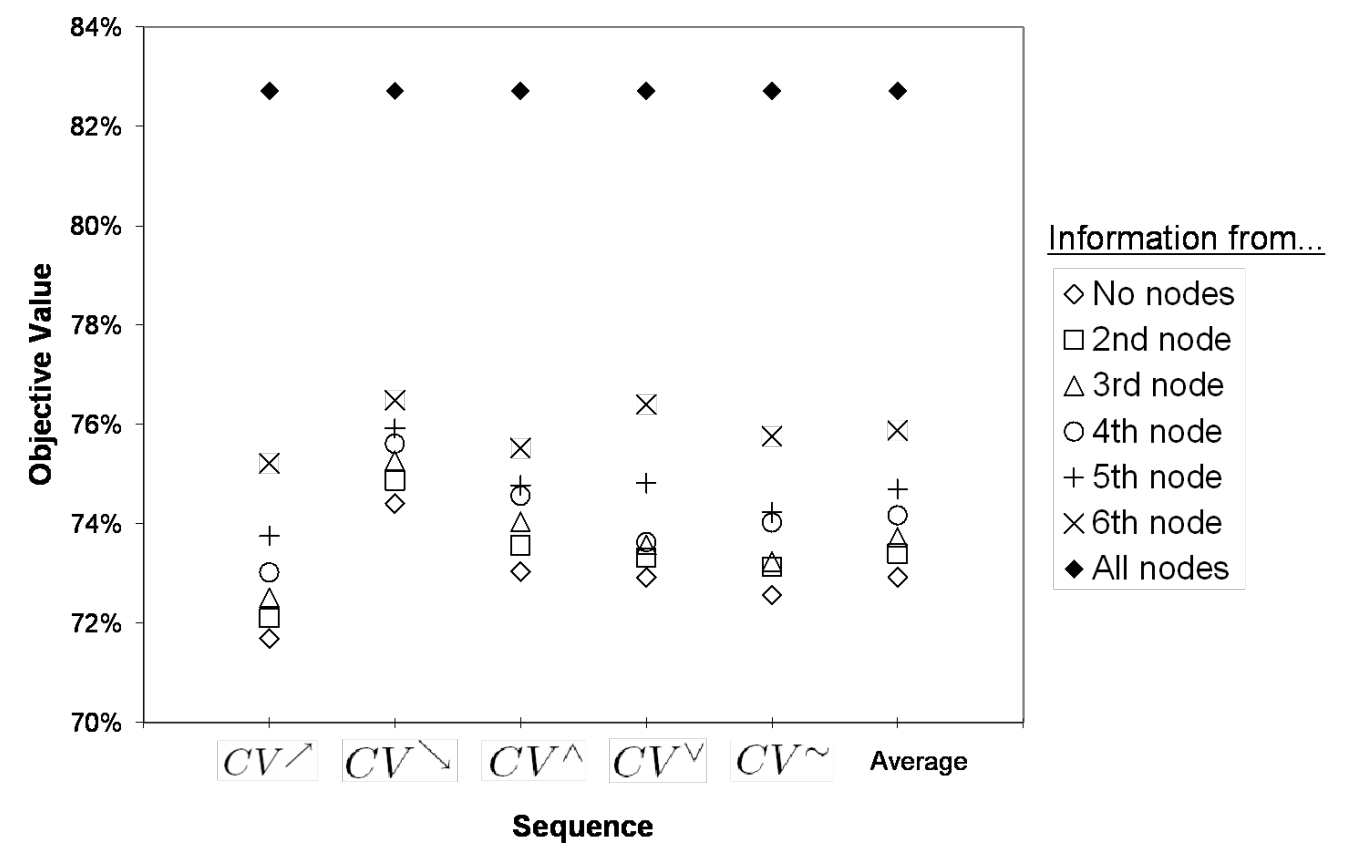

Figure 7: Average objective values for information from different node positions, node sets A-E

From Figure 7, we see that, on average, $v_{c}$ is at least $6 \%$ greater than the value of obtaining information from a single node. We define the value of partial information $\left(v_{p}\right)$, as follows:

$$
v_{p}=\frac{g_{c}-g_{p}}{v_{c}}
$$


where $v_{p}$ represents the portion of $v_{c}$ captured and where $g_{p}$ and $g_{c}$ represents the objective value for the scenario with partial and complete information, respectively. For the sixth position, $v_{p}$ is on average $30 \%$ which is greater than obtaining information from any other position. In other words, one can obtain, on average, $30 \%$ of the value of complete information if one receives information only from the last node in the sequence. Further, we are able to make the following observation:

Observation 3. In general, the value of demand information from one node $\left(v_{p}\right)$ is greater than that of another node if it is visited later in the sequence.

By this observation, it is best to obtain information from the farthest position in a route as possible. The intuition is that the information from nodes at the end of a route are useful in more decisions than information from nodes at the beginning of the route. For our six-node problem, information from the sixth node is used in decisions at the first through fifth nodes, while information at the second node is only useful in the allocation decision at the first node.

In answering question 2, from Figure 7, we also see that $v_{p}$ is greater if the node providing information has higher demand variability. For example, consider the $\searrow$ and $\wedge$ sequences (for both $\mu$ and CV), where the node with the highest variability is sequenced third. In Figure 7, we see that $v_{p}$ for the third position is greater in these two sequences than in the other three sequences. Similarly, we see that if information was obtained from the fourth position, $v_{p}$ is highest for the $\wedge$ sequence, and by contrast is lowest for the $\vee$ sequence.

To further investigate the impact of a node's demand characteristics, we consider obtaining demand information from nodes with the following: largest and smallest $\mu$, and largest and smallest $C V$. Note that for $\mu$, node sets A-D are not considered since nodes have identical $\mu$; and for CV, node sets E-H are not considered since nodes have identical CV. We present the average $v_{p}$ of each characteristic in Table 6.

\begin{tabular}{|c|cc|cc|}
\hline \multirow{2}{*}{ Sequence } & \multicolumn{4}{|c|}{ Information from node with .. } \\
\cline { 2 - 5 } & Highest $\mu$ & Lowest $\mu$ & Highest CV & Lowest CV \\
\hline \hline$\nearrow$ & $34.6 \%$ & $5.0 \%$ & $31.8 \%$ & $3.8 \%$ \\
$\searrow$ & $12.5 \%$ & $16.0 \%$ & $5.9 \%$ & $24.2 \%$ \\
$\wedge$ & $21.6 \%$ & $20.8 \%$ & $16.1 \%$ & $25.4 \%$ \\
$\vee$ & $34.0 \%$ & $9.4 \%$ & $36.3 \%$ & $6.8 \%$ \\
$\sim$ & $5.4 \%$ & $5.0 \%$ & $5.2 \%$ & $6.4 \%$ \\
\hline Total & $21.6 \%$ & $11.2 \%$ & $19.1 \%$ & $13.3 \%$ \\
\hline
\end{tabular}

Table 6: Average $v_{p}$ captured by each case 
From the results in Table 6, we make the following observation:

Observation 4. The demand characteristics of a node have an impact on its value of demand information; its value is higher if the node has higher $\mu$ or higher $C V$ relative to other nodes in the sequence.

As an example, consider the $\nearrow$ and $\searrow$ sequences. In the $\nearrow$ sequence, the nodes with the highest $\mu$ and $\mathrm{CV}$ are positioned last in the sequence; while in the $\searrow$ sequence, the nodes with the smallest $\mu$ and $\mathrm{CV}$ are positioned last in the sequence. On average, the difference of $v_{p}$ between 'Highest $\mu$ ' for $\nearrow$ and 'Lowest $\mu$ ' for $\searrow$ is $34.6 \%-16.0 \%=18.6 \%$, which implies that the last node's $\mu$ has an impact on $v_{p}$. In addition, the average difference of $v_{p}$ between 'Highest $\mu$ ' for $\searrow$ and 'Lowest $\mu$ ' for $\nearrow$ is $12.5 \%-5.0 \%=7.5 \%$; therefore, the value $v_{p}$ is higher if the demand information is from a node with higher $\mu$. Similar results hold for $\mathrm{CV}$, and for the $\wedge$ and $\vee$ seqeunces.

In conclusion, in scenarios with low $\mathrm{CV}$ and high supply the value of information is minimal, in all other scenarios the objective can be improved by obtaining demand information. The greatest impact of information is in scenarios with high CV, low supply and routed in increasing order of demand variability. The benefit may be as high as an $18.7 \%$ increase in expected minimum fill rate. If information can only be obtained from one node, the value of information is maximized if the node is as far along in the sequence as possible. The benefit from obtaining information from the last sequenced node is as high as an $7.2 \%$ increase in expected minimum fill rate. The value of information can be further improved if the selected node has higher $\mu$ or CV relative to other nodes in the sequence.

\section{Expected Waste}

In the FRP, the food distributed is perishable and considered wasted if not distributed. Along with the objective of promoting equity, minimizing waste is also a goal. In this section, we compare the expected waste under an objective that maximizes the expected minimum fill rate with that under an allocation policy that minimizes the expected waste.

We measure waste as the percentage of $s_{0}$ that is not distributed to nodes. The waste minimization allocation policy allocates the maximum possible amount at each node so to minimize the amount of undistributed supply. The expected waste under an allocation policy is calculated through a computer program that generates all sample paths of demand realizations. In Table 7, we present the average 
difference in the expected minimum fill rate and expected waste for the two policies. Let $\mathcal{Z}$ and $\omega$ represent the expected minimum fill rate and expected waste from the fill rate objective, respectively, and let $g_{W}$ and $\omega_{W}$ represent the expected minimum fill rate and expected waste from the waste objective, respectively.

\begin{tabular}{|c|cc|}
\hline \multirow{2}{*}{ Number of nodes } & Fill rate optimality gap & Expected waste optimality gap \\
\cline { 2 - 3 } & $\mathcal{Z}-g_{W}$ & $\omega-\omega_{W}$ \\
\hline 2 & $8.0 \%$ & $1.2 \%$ \\
3 & $11.9 \%$ & $1.7 \%$ \\
4 & $13.1 \%$ & $2.4 \%$ \\
5 & $14.5 \%$ & $2.7 \%$ \\
6 & $15.5 \%$ & $3.1 \%$ \\
7 & $16.3 \%$ & $3.4 \%$ \\
\hline Total & $13.2 \%$ & $2.4 \%$ \\
\hline
\end{tabular}

Table 7: Average difference in expected minimum fill rate and expected waste between fill rate and waste policies

From the results in Table 7, we make the following observation:

Observation 5. The optimal allocation policy for the objective of maximizing the expected minimum fill rates results in close-to-minimal expected waste levels.

The results show that operating under the optimal resource allocation policy results in $2.4 \%$ more expected waste, on average, then the minimal waste allocation policy. This is relatively small when compared to the poor performance in expected minimum fill rate of the waste minimization policy. Under a waste minimization policy the expected minimum fill rate is on average $13.2 \%$ worse than that of the optimal value. This is not surprising, since the waste minimization objective produces an extreme allocation policy which ignores fill rates. The optimal resource allocation policy produces only $2.4 \%$ additional waste; therefore, following the objective of maximizing the expected minimum fill rates keeps waste at a level close to the minimum.

\section{Conclusion}

In this paper we address design and operating policies of a nonprofit sequential resource allocation problem. Motivated by the goal of the GCFD to provide equitable service while maintaining a high level of distribution to its agencies, we defined the allocation objective to maximize the expected minimum fill rate. Because of the complexity of the objective, it is not possible to derive a closed form solution; further, solving for optimal allocations with dynamic programming requires more computing 
power as the size of the route increases. As a result, we developed a TND heuristic, which we have shown to be an effective approximation of the optimal allocation policy.

Further, we evaluate the objective function as it pertains to waste. Our conclusion is that under the optimal allocation policy, incurred waste is only on average less than $3 \%$ more than what is minimal. We have also studied the effects of information on the objective. Specifically, we identify scenarios in which the value of perfect demand information is negligible and scenarios in which the value is high. In the cases where it is high, the food bank and agencies should collaborate to provide demand information prior to beginning the route. Further, in the case that only one agency can provide information, it is significantly better to collect information from the last agency visited.

Throughout our analysis, we have seen that the visit sequence has an impact on fill rate. Given a set of nodes in a route, we have determined that is ideal to visit nodes in decreasing order of CV. Our model assumes that the nodes in a route are fixed. Future work can address the assignment of a larger set of agencies to multiple routes. Solving such a problem would include accounting for the distance traveled of each pickup and delivery route. Further, the selection of donors must also be considered since supply and demand along a route should be balanced.

Another extension would be to consider the problem with multiple commodities. If there is only one commodity in high demand, such as meats, then a single commodity model is sufficient since all other commodities in the program are not valued highly. In other cases, a possible objective could maximize the minimum fill rate over all commodities at an agency; which would promote equity among commodities as well as among agencies.

\section{Acknowledgment}

This research has been supported by grant CMII-0654398 from the National Science Foundation and the Northwestern University Transportation Center. The authors would also like to thank Lisa Koch, Robert Matlosz, and Eric Knepper from the Greater Chicago Food Depository.

\section{References}

Bassok, Y., R. Ernst. 1995. Dynamic allocations for multi-product distribution. Transportation Science 29(3) $256-266$.

Berman, O., R. C. Larson. 2001. Deliveries in an inventory/routing problem using a stochastic dynamic programming. Transportation Science 35(2) 192-213.

Campbell, A. M., D. Vandenbussche, W. Hermann. 2008. Routing for relief efforts. Transportation Science 42(2) $127-145$. 
Chou, M., C. Teo, H. Zheng. 2008. Process flexibility revisited: Graph expander and food-from-the-heart. Working paper, National University of Singapore Business School.

Gass, S. 1994. Public sector analysis and operations research/management science. Handbooks in Operations Research and Management Science, vol. 6. Elseiver Science B.V., 23-45.

Johnson, M. P., W. L. Gorr, S. Roehrig. 2005. Location of service facilities for the elderly. Annals of Operations Research 136(1) 329-349.

Johnson, M. P., K. R. Smilowitz. 2007. Community-based operations research. INFORMS Tutorials in Operations Research 102-123.

Kumar, A., L. B. Schwarz, J. E. Ward. 1995. Risk-pooling along a fixed delivery route using a dynamic inventory-allocation policy. Management Science 41(2) 344-362.

Mandell, M. B. 1991. Modeling effectiveness-equity trade-offs in public service delivery systems. Management Science 37(4) 467-482.

Pollock, S. M., M. H. Rothkopf, A. Barnett, eds. 1994. Operations Research and the Public Sector, Handbooks in Operations Research and Management Science, vol. 6. Elsevier Science Publishing, New York.

Savas, E. S. 1978. On equity in providing public services. Management Science 24(8) 800-806.

Savelsbergh, M.W.P., M. Sol. 1995. The general pickup and delivery problem. Transportation Science 29 17-29.

Swaminathan, J. M. 2003. Decision support for allocating scarce drugs. Interfaces 33(2) 1-11.

Wong, D. W. S., J. W. Meyer. 1993. A spatial decision support system approach to evaluate the efficiency of a meals-on-wheels program. Professional Geographer 45(3) 332-341. 


\section{ON-LINE APPENDIX A Benchmark Policy Optimality Gaps by Node Sets}

In this appendix we present the extended results of the benchmark study for resource allocation heuristics. In Table 8, we present the average and maximum optimality gaps, and the percent of cases in which the heuristic objective is within $2 \%$ of the optimal value for each node set. Results are presented for the TND, Excess Priority and Excess Sharing heuristics.

\begin{tabular}{|c|c|c|c|c|c|c|c|c|c|c|c|}
\hline & & \multicolumn{10}{|c|}{ Node set } \\
\hline & & $\mathrm{A}$ & $\mathrm{B}$ & $\mathrm{C}$ & $\mathrm{D}$ & $\mathrm{E}$ & $\mathrm{F}$ & G & $\mathrm{H}$ & I & $\bar{J}$ \\
\hline \multirow{3}{*}{$\begin{array}{l}\text { TND } \\
\text { Heuristic }\end{array}$} & Average optimality gap & $1.1 \%$ & $1.2 \%$ & $1.1 \%$ & $1.1 \%$ & $0.8 \%$ & $1.8 \%$ & $0.7 \%$ & $1.8 \%$ & $1.4 \%$ & $1.2 \%$ \\
\hline & Maximum optimality gap & $3.6 \%$ & $3.7 \%$ & $3.1 \%$ & $3.0 \%$ & $3.2 \%$ & $4.9 \%$ & $2.6 \%$ & $4.2 \%$ & $4.3 \%$ & $4.4 \%$ \\
\hline & $\%$ of cases gap $\leq 2 \%$ & $93 \%$ & $88 \%$ & $91 \%$ & $91 \%$ & $97 \%$ & $83 \%$ & $99 \%$ & $71 \%$ & $87 \%$ & $90 \%$ \\
\hline \multirow{3}{*}{$\begin{array}{l}\text { Priority } \\
\text { Mean }\end{array}$} & Average optimality gap & $6.6 \%$ & $6.6 \%$ & $6.6 \%$ & $6.7 \%$ & $6.0 \%$ & $7.3 \%$ & $5.7 \%$ & $7.4 \%$ & $6.8 \%$ & $7.2 \%$ \\
\hline & Maximum optimality gap & $12.2 \%$ & $12.4 \%$ & $11.6 \%$ & $11.5 \%$ & $12.9 \%$ & $15.5 \%$ & $12.3 \%$ & $15.5 \%$ & $14.8 \%$ & $15.2 \%$ \\
\hline & $\%$ of cases gap $\leq 2 \%$ & $5 \%$ & $5 \%$ & $2 \%$ & $2 \%$ & $11 \%$ & $10 \%$ & $11 \%$ & $8 \%$ & $10 \%$ & $10 \%$ \\
\hline \multirow{3}{*}{$\begin{array}{l}\text { Priority } \\
\text { Median }\end{array}$} & Average optimality gap & $10.8 \%$ & $10.7 \%$ & $7.7 \%$ & $6.9 \%$ & $6.0 \%$ & $7.3 \%$ & $5.7 \%$ & $6.7 \%$ & $8.5 \%$ & $15.3 \%$ \\
\hline & Maximum optimality gap & $30.0 \%$ & $30.2 \%$ & $19.6 \%$ & $16.6 \%$ & $12.9 \%$ & $15.7 \%$ & $12.3 \%$ & $13.4 \%$ & $23.5 \%$ & $37.2 \%$ \\
\hline & $\%$ of cases gap $\leq 2 \%$ & $20 \%$ & $20 \%$ & $12 \%$ & $9 \%$ & $11 \%$ & $10 \%$ & $11 \%$ & $8 \%$ & $20 \%$ & $15 \%$ \\
\hline \multirow{3}{*}{$\begin{array}{l}\text { Sharing } \\
\text { Mean }\end{array}$} & Average optimality gap & $9.2 \%$ & $9.2 \%$ & $9.2 \%$ & $9.3 \%$ & $7.2 \%$ & $10.5 \%$ & $6.9 \%$ & $10.8 \%$ & $9.0 \%$ & $10.0 \%$ \\
\hline & Maximum optimality gap & $19.3 \%$ & $19.5 \%$ & $18.6 \%$ & $18.6 \%$ & $16.6 \%$ & $23.6 \%$ & $15.3 \%$ & $21.5 \%$ & $20.4 \%$ & $23.7 \%$ \\
\hline & $\%$ of cases gap $\leq 2 \%$ & $5 \%$ & $5 \%$ & $2 \%$ & $2 \%$ & $8 \%$ & $8 \%$ & $10 \%$ & $8 \%$ & $10 \%$ & $9 \%$ \\
\hline \multirow{3}{*}{$\begin{array}{l}\text { Sharing } \\
\text { Median }\end{array}$} & Average optimality gap & $14.7 \%$ & $14.7 \%$ & $10.8 \%$ & $9.9 \%$ & $7.2 \%$ & $10.5 \%$ & $6.9 \%$ & $10.1 \%$ & $11.6 \%$ & $19.9 \%$ \\
\hline & Maximum optimality gap & $36.4 \%$ & $36.9 \%$ & $27.1 \%$ & $25.1 \%$ & $16.6 \%$ & $23.7 \%$ & $15.4 \%$ & $21.8 \%$ & $29.3 \%$ & $43.0 \%$ \\
\hline & $\%$ of cases gap $\leq 2 \%$ & $15 \%$ & $15 \%$ & $10 \%$ & $8 \%$ & $8 \%$ & $8 \%$ & $10 \%$ & $8 \%$ & $14 \%$ & $12 \%$ \\
\hline
\end{tabular}

Table 8: Optimality gaps by node sets for allocation heuristics

\section{ON-LINE APPENDIX B}

\section{Proofs of the Analytical Results}

For reference we provide a list of equations from the paper:

$$
\begin{gathered}
Z^{(i)}\left(s_{i}, \beta_{\text {min }}^{i-1}, d_{i}\right)=\max _{x_{i}} \mathbb{E}_{D_{i+1}}\left[1 \wedge \beta_{\text {min }}^{i-1} \wedge \frac{x_{i}}{d_{i}} \wedge Z^{(i+1)}\left(s_{i}-x_{i}, \beta_{m i n}^{i}, d_{i+1}\right)\right] \\
\mathcal{B}_{D_{i+1}}\left(x_{i} \mid s_{i}, \beta_{m i n}^{i-1}, d_{i}\right)=\mathbb{E}_{D_{i+1}}\left[1 \wedge \beta_{\text {min }}^{i-1} \wedge \frac{x_{i}}{d_{i}} \wedge Z^{(i+1)}\left(s_{i}-x_{i}, \beta_{m i n}^{i}, d_{i+1}\right)\right] \\
Z^{(i)}\left(s_{i}, \beta_{m i n}^{i-1}, d_{i}\right)=\max _{x_{i}} \mathcal{B}_{D_{i+1}}\left(x_{i} \mid s_{i}, \beta_{m i n}^{i-1}, d_{i}\right)
\end{gathered}
$$

Lemma 1. The family of functions $\mathcal{B}_{D_{i+1}}\left(x_{i} \mid s_{i}, \beta_{\text {min }}^{i-1}, d_{i}\right)$ is concave with respect to the allocation decision, $x_{i}$, and the supply, $s_{i}$, for $i=1,2, \ldots, N-1$.

Proof: We begin with $i=N-1$ and show that $\mathcal{B}_{D_{N}}\left(x_{N-1} \mid s_{N-1}, \beta_{m i n}^{N-2}, d_{N-1}\right)$ is concave with respect to $s_{N-1}$ and $x_{N-1}$. Then we show in an iterative manner that the function is also concave for $i=1,2, \ldots, N-2$.

For $i=N-1$, we have:

$$
\mathcal{B}_{D_{N}}\left(x_{N-1} \mid s_{N-1}, \beta_{m i n}^{N-2}, d_{N-1}\right)=\mathbb{E}_{D_{N}}\left[1 \wedge \beta_{\min }^{N-2} \wedge \frac{x_{N-1}}{d_{N-1}} \wedge \frac{s_{N-1}-x_{N-1}}{d_{N}}\right]
$$


For each realization of $d_{N}$, the function $\left(1 \wedge \beta_{\text {min }}^{N-2} \wedge \frac{x_{N-1}}{d_{N-1}} \wedge \frac{s_{N-1}-x_{N-1}}{d_{N}}\right)$ is concave with respect to $s_{N-1}$ and $x_{N-1}$. Since the linear combination of concave functions is also concave, then $\mathcal{B}_{D_{N}}\left(x_{N-1} \mid s_{N-1}, \beta_{m i n}^{N-2}, d_{N-1}\right)=$ $\mathbb{E}_{D_{N}}\left[1 \wedge \beta_{\min }^{N-2} \wedge \frac{x_{N-1}}{d_{N-1}} \wedge \frac{s_{N-1}-x_{N-1}}{d_{N}}\right]$ is concave with respect to $s_{N-1}$ and $x_{N-1}$.

We now show that $\mathcal{B}_{D_{N-1}}\left(x_{N-2} \mid s_{N-2}, \beta_{m i n}^{N-3}, d_{N-2}\right)$ is concave in $x_{N-2}$ and $s_{N-2}$. Note that with (A-3) and concavity preservation under maximization, $Z^{(N-1)}\left(s_{N-1}, \beta_{m i n}^{N-2}, d_{N-1}\right)$ is concave with respect to $s_{N-1}$. Since $s_{N-1}=s_{N-2}-x_{N-2}$, then $Z^{(N-1)}\left(s_{N-1}, \beta_{m i n}^{N-2}, d_{N-1}\right)$ is concave with respect to $x_{N-2}$ and $s_{N-2}$. Therefore, for every realization of $d_{N-1}$, the function $\left(1 \wedge \beta_{\min }^{N-2} \wedge \frac{x_{N-1}}{d_{N-1}} \wedge Z^{(N-1)}\left(s_{N-1}, \beta_{m i n}^{N-2}, d_{N-1}\right)\right)$ is concave in $x_{N-2}$ and $s_{N-2}$, as is its expectation $\mathcal{B}_{D_{N-1}}\left(x_{N-2} \mid s_{N-2}, \beta_{\text {min }}^{N-3}, d_{N-2}\right)$.

We can prove for all other nodes in an iterative manner that the functions $\mathcal{B}_{D_{i+1}}\left(x_{i} \mid s_{i}, \beta_{\text {min }}^{i-1}, d_{i}\right)$ are concave with respect to the allocation decision, $x_{i}$ and supply level, $s_{i}$.

Lemma 2. The optimal value $Z^{(i)}\left(s_{i}, \beta_{m i n}^{i-1}, d_{i}\right)$ :

(i) is non-decreasing with respect to supply, $s_{i}$; i.e., if $s_{i}^{A}>s_{i}^{B}$, then $Z^{(i)}\left(s_{i}^{A}, \beta_{m i n}^{i-1}, d_{i}\right) \geq Z^{(i)}\left(s_{i}^{B}, \beta_{m i n}^{i-1}, d_{i}\right)$.

(ii) is non-increasing with respect to demand, $d_{i}$; i.e., if $d_{i}^{A}<d_{i}^{B}$, then $Z^{(i)}\left(s_{i}, \beta_{m i n}^{i-1}, d_{i}^{A}\right) \geq Z^{(i)}\left(s_{i}, \beta_{m i n}^{i-1}, d_{i}^{B}\right)$.

Proof: We first present the proof of $(i)$ followed by the proof of $(i i)$. To prove $(i)$, let $x_{i}^{B *}$ represent the optimal allocation for supply level, $s_{i}^{B}$ at node $i$. The optimal value, $Z^{(i)}\left(s_{i}^{B}, \beta_{m i n}^{i-1}, d_{i}\right)$, is then:

$$
Z^{(i)}\left(s_{i}^{B}, \beta_{\text {min }}^{i-1}, d_{i}\right)=\mathcal{B}_{D_{i+1}}\left(x_{i}^{B *} \mid s_{i}^{B}, \beta_{\text {min }}^{i-1}, d_{i}\right)=\mathbb{E}_{D_{i+1}}\left[Z^{(i+1)}\left(s_{i}^{B}-x_{i}^{B *}, \beta_{\min }^{i-1} \wedge \frac{x_{i}^{B *}}{d_{i}}, d_{i+1}\right)\right] .
$$

For any supply level $s_{i}^{A}$, where $s_{i}^{A}>s_{i}^{B *}$, consider allocation, $x_{i}^{A}=x_{i}^{B *}+\left(s_{i}^{A}-s_{i}^{B}\right)$ at node $i$. Since $s_{i}^{A}>s_{i}^{B}$, then $x_{i}^{A}>x_{i}^{B *}$. The value of this allocation is:

$$
\mathcal{B}_{D_{i+1}}\left(x_{i}^{A} \mid s_{i}^{A}, \beta_{\text {min }}^{i-1}, d_{i}\right)=\mathbb{E}_{D_{i+1}}\left[Z^{(i+1)}\left(s_{i}^{A}-x_{i}^{A}, \beta_{\min }^{i-1} \wedge \frac{x_{i}^{A}}{d_{i}}, d_{i+1}\right)\right]
$$

Replace $s_{i}^{A}-x_{i}^{A}$ with $s_{i}^{B}-x_{i}^{B *}$ to obtain:

$$
\mathcal{B}_{D_{i+1}}\left(x_{i}^{A} \mid s_{i}^{A}, \beta_{\text {min }}^{i-1}, d_{i}\right)=\mathbb{E}_{D_{i+1}}\left[Z^{(i+1)}\left(s_{i}^{B}-x_{i}^{B *}, \beta_{\text {min }}^{i-1} \wedge \frac{x_{i}^{A}}{d_{i}}, d_{i+1}\right)\right] .
$$

From equations (A-4) and (A-6) it is clear that $\mathcal{B}_{D_{i+1}}\left(x_{i}^{A} \mid s_{i}^{A}, \beta_{m i n}^{i-1}, d_{i}\right) \geq \mathcal{B}_{D_{i+1}}\left(x_{i}^{B *} \mid s_{i}^{B}, \beta_{\text {min }}^{i-1}, d_{i}\right)$, since $x_{i}^{A}>$ $x_{i}^{B *}$. By definition $(\mathrm{A}-3), Z^{(i)}\left(s_{i}^{A}, \beta_{\text {min }}^{i-1}, d_{i}\right) \geq \mathcal{B}_{D_{i+1}}\left(x_{i}^{A} \mid s_{i}^{A}, \beta_{\text {min }}^{i-1}, d_{i}\right)$; therefore,

$$
Z^{(i)}\left(s_{i}^{A}, \beta_{\text {min }}^{i-1}, d_{i}\right) \geq \mathcal{B}_{D_{i+1}}\left(x_{i}^{A} \mid s_{i}^{A}, \beta_{\text {min }}^{i-1}, d_{i}\right) \geq \mathcal{B}_{D_{i+1}}\left(x_{i}^{B *} \mid s_{i}^{B}, \beta_{\text {min }}^{i-1}, d_{i}\right)=Z^{(i)}\left(s_{i}^{B}, \beta_{\text {min }}^{i-1}, d_{i}\right) .
$$

To prove $(i i)$, let $x_{i}^{B *}$ represent the optimal allocation for demand, $d_{i}^{B}$. We have:

$$
Z^{(i)}\left(s_{i}, \beta_{m i n}^{i-1}, d_{i}^{B}\right)=\mathcal{B}_{D_{i+1}}\left(x_{i}^{B *} \mid s_{i}, \beta_{m i n}^{i-1}, d_{i}^{B}\right)=\mathbb{E}_{D_{i+1}}\left[Z^{(i+1)}\left(s_{i}-x_{i}^{B *}, \beta_{\min }^{i-1} \wedge \frac{x_{i}^{B *}}{d_{i}^{B}}, d_{i+1}\right)\right] .
$$

Now consider the same allocation $x_{i}^{B *}$ at node $i$ for demand $d_{i}^{A}$. Because $d_{i}^{A}<d_{i}^{B}$, then $\frac{x_{i}^{B *}}{d_{i}^{A}}>\frac{x_{i}^{B *}}{d_{i}^{B}}$. The value of this allocation is:

$$
\mathcal{B}_{D_{i+1}}\left(x_{i}^{B *} \mid s_{i}, \beta_{\min }^{i-1}, d_{i}^{A}\right)=\mathbb{E}_{D_{i+1}}\left[Z^{(i+1)}\left(s_{i}-x_{i}^{B *}, \beta_{\min }^{i-1} \wedge \frac{x_{i}^{B *}}{d_{i}^{A}}, d_{i+1}\right)\right] .
$$

From equations (A-7) and (A-8) it is clear that $\mathcal{B}_{D_{i+1}}\left(x_{i}^{B *} \mid s_{i}, \beta_{\text {min }}^{i-1}, d_{i}^{A}\right) \geq \mathcal{B}_{D_{i+1}}\left(x_{i}^{B *} \mid s_{i}, \beta_{m i n}^{i-1}, d_{i}^{B}\right)$, since $\frac{x_{i}^{B *}}{d_{i}^{A}}>$ $\frac{x_{i}^{B *}}{d_{i}^{B}}$. By definition (A-3), $Z^{(i)}\left(s_{i}, \beta_{\min }^{i-1}, d_{i}^{A}\right) \geq \mathcal{B}_{D_{i+1}}\left(x_{i}^{B *} \mid s_{i}, \beta_{\min }^{i-1}, d_{i}^{A}\right) ;$ therefore,

$$
Z^{(i)}\left(s_{i}, \beta_{\text {min }}^{i-1}, d_{i}^{A}\right) \geq \mathcal{B}_{D_{i+1}}\left(x_{i}^{B *} \mid s_{i}, \beta_{\text {min }}^{i-1}, d_{i}^{A}\right) \geq \mathcal{B}_{D_{i+1}}\left(x_{i}^{B *} \mid s_{i}, \beta_{\text {min }}^{i-1}, d_{i}^{B}\right)=Z^{(i)}\left(s_{i}, \beta_{\text {min }}^{i-1}, d_{i}^{B}\right) .
$$

Hence, $Z^{(i)}\left(s_{i}, \beta_{\min }^{i-1}, d_{i}^{A}\right) \geq Z^{(i)}\left(s_{i}, \beta_{\min }^{i-1}, d_{i}^{B}\right)$. 
Theorem 1. (Structure of Optimal Resource Allocation Policy) In a two-node SRA-e problem, for a given supply $s_{1}$, the optimal allocation $x_{1}^{*}$ is a piecewise function of $d_{1}$. Specifically, there exists a threshold, $T_{d}\left(s_{1}\right)$, such that:

(i) if $d_{1} \leq T_{d}\left(s_{1}\right)$, then the optimal allocation is $x_{1}^{*}=d_{1}$.

(ii) if $d_{1}>T_{d}\left(s_{1}\right)$, then the optimal allocation is $x_{1}^{*}=H_{1}\left(s_{1}, d_{1}\right)$, where $H_{1}\left(s_{1}, d_{1}\right)<d_{1}$.

(iii) the threshold value, $T_{d}\left(s_{1}\right)$, is non-decreasing in $s_{1}$, and $H_{1}\left(s_{1}, d_{1}\right)$ is a strictly increasing concave function of $d_{1}$.

Proof: We first prove the existence of $T_{d}\left(s_{1}\right)$, which is the largest value of demand at node 1 such that $x_{1}^{*}=d_{1}$ and use this result to prove (ii). Then we show that $H_{1}\left(s_{1}, d_{1}\right)$ is a strictly increasing concave function of $d_{1}$ (as claimed in (iii)) and use this result to prove $(i)$. We end by completing the proof of (iii).

To prove the existence of $T_{d}\left(s_{1}\right)$, we show that there exists a value of demand at node $1\left(T_{d}\left(s_{1}\right)=d_{1}^{\prime}\right)$ such that (a) for $d_{1}=d_{1}^{\prime}$, then $x_{1}^{*}=d_{1}$ and (b) for $d_{1}>d_{1}^{\prime}$, then $x_{1}^{*}<d_{1}$. To do this we use the first and second order conditions of the function $\mathcal{B}_{D_{2}}\left(x_{1} \mid s_{1}, 1, d_{1}\right)$ for the case where $x_{1} \leq d_{1}$.

$$
\begin{aligned}
\mathcal{B}_{D_{2}}\left(x_{1} \mid s_{1}, 1, d_{1}\right) & =\mathbb{E}_{D_{2}}\left[\frac{x_{1}}{d_{1}} \wedge \frac{s_{1}-x_{1}}{D_{2}}\right] \\
& =\int_{0}^{\frac{d_{1}}{x_{1}}\left(s_{1}-x_{1}\right)} \frac{x_{1}}{d_{1}} f\left(d_{2}\right) d d_{2}-\int_{\frac{d_{1}}{x_{1}}\left(s_{1}-x_{1}\right)}^{\infty} \frac{s_{1}-x_{1}}{d_{2}} f\left(d_{2}\right) d d_{2}
\end{aligned}
$$

where $f\left(d_{2}\right)\left(F\left(d_{2}\right)\right)$ represent the probability (cumulative) density function of demand at node $2 . \mathcal{B}_{D_{2}}\left(x_{1} \mid s_{1}, 1, d_{1}\right)$ is maximized at $x_{1}^{*}$ which satisfies the following first order condition:

$$
W\left(x_{1}^{*}\right)=\int_{0}^{\frac{d_{1}}{x_{1}^{*}}\left(s_{1}-x_{1}^{*}\right)} \frac{1}{d_{1}} f\left(d_{2}\right) d d_{2}-\int_{\frac{d_{1}}{x_{1}^{*}}\left(s_{1}-x_{1}^{*}\right)}^{\infty} \frac{1}{d_{2}} f\left(d_{2}\right) d d_{2}=0 .
$$

The solution to (A-10) is a maximum since the second derivative is clearly negative:

$$
\frac{d W\left(x_{1}^{*}\right)}{d x_{1}}=-\frac{s_{1}}{d_{1} x_{1}} f\left(\frac{d_{1}}{x_{1}}\left(s_{1}-x_{1}\right)\right) \frac{d_{1}}{x_{1}} \frac{s_{1}}{s_{1}-x_{1}} .
$$

To show that there exists $T_{d}\left(s_{1}\right)$ we must show that $W\left(x_{1}^{*}=T_{d}\left(s_{1}\right)\right)=0$ for some value of $d_{1}=T_{d}\left(s_{1}\right)$. With $x_{1}^{*}=d_{1}=T_{d}\left(s_{1}\right),(\mathrm{A}-10)$ becomes:

$$
W\left(x_{1}^{*}=T_{d}\left(s_{1}\right)\right)=\int_{0}^{\left(s_{1}-T_{d}\left(s_{1}\right)\right)} \frac{1}{T_{d}\left(s_{1}\right)} f\left(d_{2}\right) d d_{2}-\int_{\left(s_{1}-T_{d}\left(s_{1}\right)\right)}^{\infty} \frac{1}{d_{2}} f\left(d_{2}\right) d d_{2}
$$

By definition, $x_{1}^{*}=T_{d}\left(s_{1}\right) \in\left(0, s_{1}\right]$. Note that $W\left(T_{d}\left(s_{1}\right)\right) \rightarrow \infty$ as $T_{d}\left(s_{1}\right) \rightarrow 0$ and $W\left(T_{d}\left(s_{1}\right)=s_{1}\right)<0$. Further,

$$
\frac{d W\left(T_{d}\left(s_{1}\right)\right)}{d T_{d}\left(s_{1}\right)}=-\left[\frac{1}{T_{d}\left(s_{1}\right)^{2}} F\left(s_{1}-T_{d}\left(s_{1}\right)\right)+\frac{s_{1}}{T_{d}\left(s_{1}\right)\left(s_{1}-T_{d}\left(s_{1}\right)\right)} f\left(s_{1}-T_{d}\left(s_{1}\right)\right)\right]<0
$$

for all $T_{d}\left(s_{1}\right) \in\left(0, s_{1}\right]$; therefore, $W\left(T_{d}\left(s_{1}\right)\right)$ is continuously decreasing with respect to $T_{d}\left(s_{1}\right)$ and $W\left(T_{d}\left(s_{1}\right)\right)=0$ at a unique point. We conclude that there exists a unique $T_{d}\left(s_{1}\right)$ such that if $d_{1}=T_{d}\left(s_{1}\right)$, then $x_{1}^{*}=d_{1}$. Since (A-9) holds only for $x_{1} \leq d_{1}$ and $T_{d}\left(s_{1}\right)$ is unique, then $T_{d}\left(s_{1}\right)$ is the largest value of demand at node 1 such that $x_{1}^{*}=d_{1}$. Therefore, $x_{1}^{*}<d_{1}$ for $d_{1}>T_{d}\left(s_{1}\right)$, as claimed in $(i i)$.

Next we prove $H_{1}\left(s_{1}, d_{1}\right)$ is a strictly increasing concave function of $d_{1}$ and use this result to prove $(i)$. Consider $d_{1} \geq T_{d}\left(s_{1}\right)$, thus $x_{1}^{*} \leq d_{1}$. The value of allocation $x_{1}$ is found by (A-9), and is maximized at $x_{1}^{*}$ which satisfies (A-10). To prove that $x_{1}^{*}=H_{1}\left(s_{1}, d_{1}\right)$ is a strictly increasing concave function of $d_{1}$, we show that at every value of $d_{1} \geq T_{d}\left(s_{1}\right)$ the magnitude of change in $W\left(x_{1}^{*}\right)$ with respect to $d_{1}$ is greater than it is with respect to $x_{1}^{*}$. Therefore, as demand increases, the optimal allocation increases at a lesser rate and is concave. 
Taking the derivative of $(\mathrm{A}-10)$ with respect to $d_{1}$ and $x_{1}^{*}$ results in the following equations:

$$
\begin{aligned}
& \frac{d W\left(x_{1}^{*}\right)}{d d_{1}}=\frac{s_{1}}{d_{1} x_{1}^{*}} f\left(\frac{d_{1}}{x_{1}^{*}}\left(s_{1}-x_{1}^{*}\right)\right)-\frac{1}{d_{1}^{2}} F\left(\frac{d_{1}}{x_{1}^{*}}\left(s_{1}-x_{1}^{*}\right)\right) \\
& \frac{d W\left(x_{1}^{*}\right)}{d x_{1}^{*}}=-\frac{s_{1}}{d_{1} x_{1}^{*}} f\left(\frac{d_{1}}{x_{1}^{*}}\left(s_{1}-x_{1}^{*}\right)\right) \frac{d_{1}}{x_{1}^{*}} \frac{s_{1}}{s_{1}-x_{1}^{*}}
\end{aligned}
$$

Since $d_{1} \geq x_{1}^{*} \geq 0$, then $\left|\frac{d W\left(x_{1}^{*}\right)}{d x_{1}^{*}}\right| \geq\left|\frac{d W\left(x_{1}^{*}\right)}{d d_{1}}\right|$, proving our claim.

To prove $(i)$, we have shown that for $d_{1} \geq x_{1}^{*}$, as demand increases, the optimal allocation increases at a lesser rate. Therefore, since $x_{1}^{*}=d_{1}$ for $d_{1}=T_{d}\left(s_{1}\right)$, the optimal allocation for $d_{1}<T_{d}\left(s_{1}\right)$ cannot be less than $d_{1}$; i.e., $x_{1}^{*} \geq d_{1}$. By definition, allocations cannot be more than demand; therefore, $x_{1}^{*}=d_{1}$ for $d_{1} \leq T_{d}\left(s_{1}\right)$.

Finally, we complete the proof of $(i i i)$. We treat $T_{d}\left(s_{1}\right)$ independently of $s_{1}$ and take the derivative of $W\left(T_{d}\left(s_{1}\right)\right)$ with respect to $s_{1}$, which is

$$
\frac{d W\left(T_{d}\left(s_{1}\right)\right)}{d\left(s_{1}\right)}=\frac{s_{1}}{T_{d}\left(s_{1}\right)\left(s_{1}-T_{d}\left(s_{1}\right)\right)} f\left(s_{1}-T_{d}\left(s_{1}\right)\right)>0 .
$$

Therefore, since $\frac{d W\left(T_{d}\left(s_{1}\right)\right)}{d T_{d}\left(s_{1}\right)}<0$, as $s_{1}$ increases, $T_{d}\left(s_{1}\right)$ increases so that $W\left(T_{d}\left(s_{1}\right)\right)=0$ holds, proving that $T_{d}\left(s_{1}\right)$ is non-decreasing in $s_{1}$. At this beginning of the proof of Theorem 1 , we showed that $H_{1}\left(s_{1}, d_{1}\right)$ is a strictly increasing concave function of $d_{1}$.

Theorem 2. (Sensitivity of the Optimal Resource Allocation Policy to Supply) In a two-node SRA-e problem, for a given demand $d_{1}$ there exists a supply level, $T_{s}\left(d_{1}\right)$, such that:

(i) for $s_{1}<T_{s}\left(d_{1}\right)$, the optimal allocation, $x_{1}^{*}=H_{1}\left(s_{1}, d_{1}\right)<d_{1}$, where $H_{1}\left(s_{1}, d_{1}\right)$ is an increasing linear function of $s_{1}$ (i.e., $\left.H_{1}\left(s_{1}, d_{1}\right)=\alpha s_{1}\right)$. Furthermore, the optimal objective function value at node 1 , $Z^{(1)}\left(s_{1}, 1, d_{1}\right)$ is increasing with supply $s_{1}$ at a constant ratio, $\theta$.

(ii) for $s_{1} \geq T_{s}\left(d_{1}\right)$, the optimal allocation, $x_{1}^{*}=d_{1}$.

(iii) $T_{s}\left(d_{1}\right), \alpha$ and $\theta$ are non-decreasing in $d_{1}$.

Proof: We first prove the existence of $T_{s}\left(d_{1}\right)$, which is the smallest value of supply available at node 1 such that $x_{1}^{*}=d_{1}$. We use this result to prove the claims in $(i),(i i)$, and (iii).

To prove the existence of $T_{s}\left(d_{1}\right)$, we show that there exists a value of supply $\left(T_{s}\left(d_{1}\right)=s_{1}^{\prime}\right)$ such that (a) for $s_{1}=s_{1}^{\prime}$, then $x_{1}^{*}=d_{1}$ and (b) for $s_{1}<s_{1}^{\prime}$, then $x_{1}^{*}<d_{1}$. To do this, we use the first and second order conditions of the function $\mathcal{B}_{D_{2}}\left(x_{1} \mid s_{1}, 1, d_{1}\right)$ for the case where $x_{1} \leq d_{1}$.

$$
\begin{aligned}
\mathcal{B}_{D_{2}}\left(x_{1} \mid s_{1}, 1, d_{1}\right) & =\mathbb{E}_{D_{2}}\left[\frac{x_{1}}{d_{1}} \wedge \frac{s_{1}-x_{1}}{D_{2}}\right] \\
& =\int_{0}^{\frac{d_{1}}{x_{1}}\left(s_{1}-x_{1}\right)} \frac{x_{1}}{d_{1}} f\left(d_{2}\right) d d_{2}-\int_{\frac{d_{1}}{x_{1}}\left(s_{1}-x_{1}\right)}^{\infty} \frac{s_{1}-x_{1}}{d_{2}} f\left(d_{2}\right) d d_{2}
\end{aligned}
$$

where $f\left(d_{2}\right)\left(F\left(d_{2}\right)\right)$ represent the probability (cumulative) density function of demand at node $2 . \mathcal{B}_{D_{2}}\left(x_{1} \mid s_{1}, 1, d_{1}\right)$ is maximized at $x_{1}^{*}$ which satisfies the following first order condition:

$$
W\left(x_{1}^{*}\right)=\int_{0}^{\frac{d_{1}}{x_{1}^{*}}\left(s_{1}-x_{1}^{*}\right)} \frac{1}{d_{1}} f\left(d_{2}\right) d d_{2}-\int_{\frac{d_{1}}{x_{1}^{*}}\left(s_{1}-x_{1}^{*}\right)}^{\infty} \frac{1}{d_{2}} f\left(d_{2}\right) d d_{2}=0 .
$$

The solution to (A-10) is a maximum since the second derivative is clearly negative:

$$
\frac{d W\left(x_{1}^{*}\right)}{d x_{1}}=-\frac{s_{1}}{d_{1} x_{1}} f\left(\frac{d_{1}}{x_{1}}\left(s_{1}-x_{1}\right)\right) \frac{d_{1}}{x_{1}} \frac{s_{1}}{s_{1}-x_{1}} .
$$


To show that there exists $T_{s}\left(d_{1}\right)$ we must show that $W\left(x_{1}^{*}=d_{1}\right)=0$ for some supply value $s_{1}=T_{s}\left(d_{1}\right)$. With $x_{1}^{*}=d_{1}$ and $s_{1}=T_{s}\left(d_{1}\right),(\mathrm{A}-10)$ becomes:

$$
W\left(x_{1}^{*}=T_{d}\left(s_{1}\right)\right)=\int_{0}^{\left(s_{1}-T_{d}\left(s_{1}\right)\right)} \frac{1}{T_{d}\left(s_{1}\right)} f\left(d_{2}\right) d d_{2}-\int_{\left(s_{1}-T_{d}\left(s_{1}\right)\right)}^{\infty} \frac{1}{d_{2}} f\left(d_{2}\right) d d_{2}
$$

Under these assumptions, $T_{s}\left(d_{1}\right) \geq x_{1}^{*}=d_{1}$, since allocation is required to be feasible. Note that if $T_{s}\left(d_{1}\right)=\infty$ then $W\left(d_{1}\right)>0$, and if $T_{s}\left(d_{1}\right)=d_{1}$ then $W\left(d_{1}\right)<0$. Further,

$$
\frac{d W\left(d_{1}\right)}{d T_{s}\left(d_{1}\right)}=\frac{T_{s}\left(d_{1}\right)}{d_{1}\left(T_{s}\left(d_{1}\right)-d_{1}\right)} f\left(T_{s}\left(d_{1}\right)-d_{1}\right)>0
$$

for all $T_{s}\left(d_{1}\right) \geq d_{1}$; therefore, $W\left(x_{1}^{*}=d_{1}\right)$ is strictly increasing as $T_{s}\left(d_{1}\right)$ increases and there exists a unique $T_{s}\left(d_{1}\right)$ such that $W\left(x_{1}^{*}=d_{1}\right)=0$.

To prove $(i)$, consider $s_{1}<T_{s}\left(d_{1}\right)$ and let $\alpha=\frac{x_{1}}{s_{1}}$ represent the ratio between allocation and supply, and rewrite (A-15) in terms of $\alpha$ :

$$
\mathcal{B}_{D_{2}}\left(\alpha s_{1} \mid s_{1}, 1, d_{1}\right)=s_{1} \mathbb{E}_{D_{2}}\left[\frac{\alpha}{d_{1}} \wedge \frac{1-\alpha}{D_{2}}\right]
$$

From (A-19) we see that optimizing $\alpha$ is independent of $s_{1}$ for $s_{1}<T_{s}\left(d_{1}\right)$. Therefore, $x_{1}^{*}$ is increasing with supply at a constant ratio. Further, from (A-19), the functions $\mathcal{B}_{D_{2}}\left(\alpha s_{1} \mid s_{1}, 1, d_{1}\right)$ and $Z^{(1)}\left(s_{1}, 1, d_{1}\right)$ given $d_{1}$ are increasing with supply at a constant ratio $\theta$, where $\theta=\mathbb{E}_{D_{2}}\left[\frac{\alpha}{d_{1}} \wedge \frac{1-\alpha}{D_{2}}\right]$.

To prove $(i i)$, we have shown that $x_{1}^{*}$ is increasing with $s_{1}$ at a constant ratio for the condition $s_{1} \leq d_{1}$. Therefore, since $x_{1}^{*}=d_{1}$ for $s_{1}=T_{s}\left(d_{1}\right)$, the optimal allocation for $s_{1}>T_{s}\left(d_{1}\right)$ cannot be less than $d_{1}$; i.e., $x_{1}^{*} \geq d_{1}$. By definition, allocations cannot be more than demand; therefore, $x_{1}^{*}=d_{1}$ for $s_{1} \geq T_{s}\left(d_{1}\right)$.

Lastly, part (iii) is proved by Theorem 1 , since $T_{s}\left(d_{1}\right)$ is an optimal allocation value for $d_{1}$. The ratio $\theta$ is non-decreasing in $d_{1}$ since $\alpha=\frac{x_{1}}{s_{1}}$ is non-decreasing in $d_{1}$.

Proposition 1. Given two nodes following the demand distributions in Figure 5(left), there exists a supply $s^{\prime}$, such that if $s_{0} \leq s^{\prime}$, increasing the demand variability of node 1 (by decreasing $p_{1}$ ) increases the expected minimum fill rate, where

$$
s^{\prime}=\frac{2 \mu(2 \mu+k)\left(p_{2} \mu+k\right)}{\left(1+p_{2}\right)(\mu+k)^{2}-\left(1-p_{2}\right) \mu^{2}} .
$$

Proof: To prove this theorem we derive the optimal allocation policy for each demand level at node 1, and then use the policy in constructing an expression for the optimal objective value. From this expression we can show that the objective is increasing as variability at node 1 increases.

First we find the optimal allocation policy for the problem in which nodes observe demand from distributions described in Figure 5(left). The details of the derivation are presented at the end of this Appendix.

The optimal allocation policy at the first node is as follows:

If $d_{1}=\mu+k$, then the optimal allocation is $x_{1}^{*}=(\mu+k) \wedge\left(s \frac{\mu+k}{2 \mu+k}\right)$.

If $d_{1}=\mu$, then the optimal allocation is $x_{1}^{*}=\mu \wedge \frac{1}{2} s$.

If $d_{1}=\mu-k$, then the optimal allocation is $x_{1}^{*}=\left\{\begin{array}{lll}\mu-k \wedge s \frac{\mu-k}{2 \mu-k} & : & \text { if } \quad p_{2} \geq \frac{\mu k}{\mu^{2}+\mu k-k^{2}} \\ \mu-k \wedge \frac{1}{2} s & : \quad \text { if } \quad p_{2}<\frac{\mu k}{\mu^{2}+\mu k-k^{2}}\end{array}\right.$

The inequality, $p_{2} \geq \frac{\mu k}{\mu^{2}+\mu k-k^{2}}$, relates the probabilities of demand observations to the ratio of $k$ to $\mu$. As this ratio increases (decreases) the right hand side of the inequality increases (decreases). Since we assume that $p_{i} \geq \frac{1-p_{i}}{2}$, then $p_{1}, p_{2} \geq \frac{1}{3}$, and a sufficient condition for $p_{2} \leq \frac{\mu k}{\mu^{2}+\mu k-k^{2}}$ is $k \geq \frac{5}{12} \mu$. 
With the optimal allocation policy above, the expected optimal objective value, $\mathcal{Z}(s)$, can be derived for each value of $s$. First consider $p_{2} \geq \frac{\mu k}{\mu^{2}+\mu k-k^{2}}$.

$$
\begin{aligned}
\mathcal{Z}(s \geq 2(\mu+k))= & 1 \\
\mathcal{Z}(2(\mu+k) \geq s \geq 2 \mu+k)= & \frac{1-p_{1}}{2}\left[\frac{1+p_{2}}{2}+\frac{s-\mu-k}{\mu+k} \frac{1-p_{2}}{2}\right]+\frac{1+p_{1}}{2} \\
\mathcal{Z}(2 \mu+k \geq s \geq 2 \mu)= & \frac{1-p_{1}}{2}\left[\frac{1+p_{2}}{2} \frac{s}{2 \mu+k}+\frac{1-p_{2}}{2} \frac{s \mu}{(2 \mu+k)(\mu+k)}\right] \\
& +p_{1}\left[\frac{1+p_{2}}{2}+\frac{1-p_{2}}{2} \frac{s-\mu}{\mu+k}\right]+\frac{1-p_{1}}{2} \\
\mathcal{Z}(2 \mu \geq s \geq 2 \mu-k)= & \frac{1-p_{1}}{2}\left[\frac{1+p_{2}}{2} \frac{s}{2 \mu+k}+\frac{1-p_{2}}{2} \frac{s \mu}{(2 \mu+k)(\mu+k)}\right] \\
& +p_{1}\left[\frac{1+p_{2}}{2} \frac{s}{2 \mu}+\frac{1-p_{2}}{2} \frac{s}{2(\mu+k)}\right]+\frac{1-p_{1}}{2}\left[\frac{1+p_{2}}{2}+\frac{1-p_{2}}{2} \frac{s-\mu+k}{\mu+k}\right] \\
\mathcal{Z}(2 \mu-k \geq s)= & \frac{1-p_{1}}{2}\left[\frac{1+p_{2}}{2} \frac{s}{2 \mu+k}+\frac{1-p_{2}}{2} \frac{s \mu}{(2 \mu+k)(\mu+k)}\right] \\
& +p_{1}\left[\frac{1+p_{2}}{2} \frac{s}{2 \mu}+\frac{1-p_{2}}{2} \frac{s}{2(\mu+k)}\right] \\
& +\frac{1-p_{1}}{2}\left[\frac{1+p_{2}}{2} \frac{s}{2 \mu-k}+\frac{1-p_{2}}{2} \frac{s \mu}{(2 \mu-k)(\mu+k)}\right]
\end{aligned}
$$

For $s \geq 2 \mu-k$, the objective values for $p_{2} \leq \frac{\mu k}{\mu^{2}+\mu k-k^{2}}$ are the same as above. For $s \leq 2 \mu-k$, the objective values are:

$$
\begin{aligned}
\mathcal{Z}(2 \mu-k \geq s \geq 2(\mu-k))= & \frac{1-p_{1}}{2}\left[\frac{1+p_{2}}{2} \frac{s}{2 \mu+k}+\frac{1-p_{2}}{2} \frac{s \mu}{(2 \mu+k)(\mu+k)}\right] \\
& +p_{1}\left[\frac{1+p_{2}}{2} \frac{s}{2 \mu}+\frac{1-p_{2}}{2} \frac{s}{2(\mu+k)}\right] \\
& +\frac{1-p_{1}}{2}\left[\frac{1-p_{2}}{2}+p_{2} \frac{s-\mu+k}{\mu}+\frac{1-p_{2}}{2} \frac{s-\mu+k}{\mu+k}\right] \\
\mathcal{Z}(2(\mu-k) \geq s)= & \frac{1-p_{1}}{2}\left[\frac{1+p_{2}}{2} \frac{s}{2 \mu+k}+\frac{1-p_{2}}{2} \frac{s \mu}{(2 \mu+k)(\mu+k)}\right] \\
& +p_{1}\left[\frac{1+p_{2}}{2} \frac{s}{2 \mu}+\frac{1-p_{2}}{2} \frac{s}{2(\mu+k)}\right] \\
& +\frac{1-p_{1}}{2}\left[\frac{1-p_{2}}{2} \frac{s}{2(\mu-k)}+p_{2} \frac{s}{2 \mu}+\frac{1-p_{2}}{2} \frac{s}{2(\mu+k)}\right]
\end{aligned}
$$

To prove the proposition, we show that if

$$
s \leq \frac{2 \mu(2 \mu+k)\left(p_{2} \mu+k\right)}{\left(1+p_{2}\right)(\mu+k)^{2}-\left(1-p_{2}\right) \mu^{2}}, \quad \text { then } \quad \frac{d \mathcal{Z}(s)}{d p_{1}} \leq 0 .
$$

Therefore $\mathcal{Z}(s)$ increases as $p_{1}$ decreases (variability increases). We only consider scenarios where $s \leq 2 \mu$, since:

$$
s^{\prime}=\frac{2 \mu(2 \mu+k)\left(p_{2} \mu+k\right)}{\left(1+p_{2}\right)(\mu+k)^{2}-\left(1-p_{2}\right) \mu^{2}} \leq 2 \mu .
$$

Case I: $p_{2} \geq \frac{\mu k}{\mu^{2}+\mu k-k^{2}}$. For $s \leq 2 \mu-k$, with some algebra

$$
\frac{d \mathcal{Z}(s)}{d p_{1}}=\frac{s}{2}\left[\frac{1+p_{2}}{2} \frac{-k^{2}}{\mu(2 \mu+k)(2 \mu-k)}+\frac{1-p_{2}}{2} \frac{-k^{2}}{(\mu+k)(2 \mu+k)(2 \mu-k)}\right],
$$

which is clearly negative.

For $2 \mu \geq s \geq 2 \mu-k$, then

$$
\frac{d \mathcal{Z}(s)}{d p_{1}}=\frac{1+p_{2}}{2}\left[\frac{s}{\mu}-\frac{2 \mu+k+s}{2 \mu+k}\right]+\frac{1-p_{2}}{2}\left[\frac{s}{\mu+k}-\frac{s \mu+(s-\mu+k)(2 \mu+k)}{(2 \mu+k)(\mu+k)}\right],
$$


which is negative for

$$
s \leq \frac{2 \mu(2 \mu+k)\left(p_{2} \mu+k\right)}{\left(1+p_{2}\right)(\mu+k)^{2}-\left(1-p_{2}\right) \mu^{2}} .
$$

Case II: $p_{2} \leq \frac{\mu k}{\mu^{2}+\mu k-k^{2}}$. For $s \leq 2(\mu-k)$,

$$
\frac{d \mathcal{Z}(s)}{d p_{1}}=\frac{s k}{4 \mu(2 \mu+k)}\left[\frac{1-p_{2}}{2} \frac{-k(3 \mu+k)}{\mu(\mu-k)(\mu+k)+p_{2}}\right],
$$

which is negative since $p_{2} \leq \frac{\mu k}{\mu^{2}+\mu k-k^{2}}$.

For $2 \mu-k \geq s \geq 2(\mu-k)$, then

$$
\frac{d \mathcal{Z}(s)}{d p_{1}}=\frac{1+p_{2}}{2}\left[\frac{-2 \mu}{2 \mu+k}+2 \mu-k 2 \mu\right]+\frac{1-p_{2}}{2}\left[\frac{-k^{2}}{2(\mu+k)(2 \mu+k)}\right],
$$

which is clearly negative.

For $2 \mu \geq s \geq 2 \mu-k$ then

$$
\frac{d \mathcal{Z}(s)}{d p_{1}}=\frac{1+p_{2}}{2}\left[\frac{s}{\mu}-\frac{2 \mu+k+s}{2 \mu+k}\right]+\frac{1-p_{2}}{2}\left[\frac{s}{\mu+k}-\frac{s \mu+(s-\mu+k)(2 \mu+k)}{(2 \mu+k)(\mu+k)}\right],
$$

which is negative for

$$
s \leq \frac{2 \mu(2 \mu+k)\left(p_{2} \mu+k\right)}{\left(1+p_{2}\right)(\mu+k)^{2}-\left(1-p_{2}\right) \mu^{2}} .
$$

Theorem 3. In a two-node SRA-e problem in which two nodes observe demand from the same family of distributions with different means, but with identical standard deviations, visiting the nodes in increasing order of demand mean will result in a higher expected minimum fill rate.

Proof: Let $L$ denote the node with the lower demand mean, and $f_{L}(\cdot)$ be its demand probability density function. Similarly, let $H$ and $f_{H}(\cdot)$ represent the same for the node with higher demand mean. Let $\Delta$ be the difference between the nodes' demand means. Thus for every demand $\epsilon$ at node $H$ there is a corresponding demand $\epsilon-\Delta$ at node $L$ so that $f_{L}(\epsilon-\Delta)=f_{H}(\epsilon)$.

To prove the theorem, for a given supply $(s)$, we show that for each pair of corresponding demands $(\epsilon-\Delta, \epsilon)$, the value of sequencing $L$ first with a demand of $\epsilon-\Delta\left(Z_{L \rightarrow H}(s, 1, \epsilon-\Delta)\right.$ is greater than or equal to the value of sequencing $H$ first with a demand of $\epsilon\left(Z_{H \rightarrow L}(s, 1, \epsilon)\right)$. Because the probabilities of the corresponding demands are identical $\left(f_{L}(\epsilon-\Delta)=f_{H}(\epsilon)\right)$, we can conclude that the relationship also holds in expectation, or in other words the expected value of sequencing $L$ first is greater than the expected value of sequencing $H$ first $\left(\mathcal{Z}_{L \rightarrow H}(s) \geq \mathcal{Z}_{H \rightarrow L}(s)\right)$.

To prove $Z_{L \rightarrow H}(s, 1, \epsilon-\Delta) \geq Z_{H \rightarrow L}(s, 1, \epsilon)$, we construct a suboptimal allocation policy for the sequence $L \rightarrow H$, based on the optimal allocation policy $\left(x_{\epsilon}^{*}\right)$ for the sequence $H \rightarrow L$. We show that under the constructed suboptimal allocation policy, the objective value of the sequence $L \rightarrow H$ is greater or equal to that of the optimal objective value for the sequence $H \rightarrow L$.

First, consider the maximization problem $Z_{H \rightarrow L}(s, 1, \epsilon)$ for visiting the higher mean demand node first. Let $x_{\epsilon}^{*}$ be the optimal allocation at the first node $(H)$ for demand $\epsilon$, thus:

$$
Z_{H \rightarrow L}(s, 1, \epsilon)=\mathcal{B}_{L}\left(x_{\epsilon}^{*} \mid s, 1, \epsilon\right)=\int_{0}^{\epsilon \frac{s-x_{*}^{*}}{x_{\epsilon}^{*}}} \frac{x_{\epsilon}^{*}}{\epsilon} f_{L}(d) d d+\int_{\epsilon \frac{s-x_{\epsilon}^{*}}{x_{\epsilon}^{*}}}^{\infty} \frac{s-x_{\epsilon}^{*}}{d} f_{L}(d) d d
$$

For the maximization problem of visiting the lower mean demand node first, $Z_{L \rightarrow H}(s, 1, \epsilon-\Delta)$, consider the value, $\mathcal{B}_{H}\left(\frac{\epsilon-\Delta}{\epsilon} x_{\epsilon}^{*} \mid s, 1, \epsilon-\Delta\right)$, of the (suboptimal) allocation $\frac{\epsilon-\Delta}{\epsilon} x_{\epsilon}^{*}$ :

$$
Z_{L \rightarrow H}(s, 1, \epsilon-\Delta) \geq \mathcal{B}_{H}\left(\frac{\epsilon-\Delta}{\epsilon} x_{\epsilon}^{*} \mid s, 1, \epsilon-\Delta\right)=\int_{0}^{\epsilon \frac{s-x_{\epsilon}^{*}}{x_{\epsilon}^{*}}+\Delta} \frac{x_{\epsilon}^{*}}{\epsilon} f_{H}(d) d d+\int_{\epsilon \frac{s-x_{\epsilon}^{*}}{x_{\epsilon}^{*}}+\Delta}^{\infty} \frac{s-\frac{\epsilon-\Delta}{\epsilon} x_{\epsilon}^{*}}{d} f_{H}(d) d d
$$


which can be expressed in terms of $f_{L}(\cdot)$, with a simple transformation:

$$
\mathcal{B}_{H}\left(\frac{\epsilon-\Delta}{\epsilon} x_{\epsilon}^{*} \mid s, 1, \epsilon-\Delta\right)=\int_{0}^{\epsilon \frac{s-x_{\epsilon}^{*}}{x_{\epsilon}^{*}}} \frac{x_{\epsilon}^{*}}{\epsilon} f_{L}(d) d d+\int_{\epsilon \frac{s-x_{\epsilon}^{*}}{x_{\epsilon}^{*}}}^{\infty} \frac{s-\frac{\epsilon-\Delta}{\epsilon} x_{\epsilon}^{*}}{d+\Delta} f_{L}(d) d d
$$

The difference between this value and $Z_{H \rightarrow L}(s, 1, \epsilon)$ can be easily derived and shown to be positive.

$$
\mathcal{B}_{H}\left(\frac{\epsilon-\Delta}{\epsilon} x_{\epsilon}^{*} \mid s, 1, \epsilon-\Delta\right)-\mathcal{B}_{L}\left(x_{\epsilon}^{*} \mid s, 1, \epsilon\right)=\int_{\epsilon \frac{s-x_{\epsilon}^{*}}{x_{\epsilon}^{*}}}^{\infty}\left(\frac{s-\frac{\epsilon-\Delta}{\epsilon} x_{\epsilon}^{*}}{d+\Delta}-\frac{s-x_{\epsilon}^{*}}{d}\right) f_{L}(d) d d \geq 0
$$

Therefore, $Z_{L \rightarrow H}(s, 1, \epsilon-\Delta) \geq \mathcal{B}_{H}\left(\frac{\epsilon-\Delta}{\epsilon} x_{\epsilon}^{*} \mid s, 1, \epsilon-\Delta\right) \geq \mathcal{B}_{L}\left(x_{\epsilon}^{*} \mid s, 1, \epsilon\right)=Z_{H \rightarrow L}(s, 1, \epsilon)$ for every demand level $\epsilon$, and thus in expectation, $\mathcal{Z}_{L \rightarrow H}(s) \geq \mathcal{Z}_{H \rightarrow L}(s)$ as desired.

Theorem 4. In a two-node SRA-e problem in which two nodes follow the demand distributions in Figure 5(left), if $p_{1}<p_{2}$, then the expected minimum fill rate is greater when node 1 (which has a higher standard deviation) is visited first.

Proof: To prove the theorem, we compare the optimal objective values of both sequences by using the optimal value of the sequence $1 \rightarrow 2, \mathcal{Z}_{1 \rightarrow 2}(s)$, as derived in the proof of Proposition 1 . The optimal values for the sequence $2 \rightarrow 1, \mathcal{Z}_{1 \rightarrow 2}(s)$, are found by exchanging $p_{1}$ and $p_{2}$ for the equations for $\mathcal{Z}_{1 \rightarrow 2}(s)$.

Assume $p_{2} \geq p_{1}$, so that node 1 has more variable demand. We show that the expected minimum fill rate visiting node 1 first is higher than that of node 2 , specifically $\mathcal{Z}_{1 \rightarrow 2}(s)-\mathcal{Z}_{2 \rightarrow 1}(s) \geq 0$. There are three demand scenarios depending on the values of $p_{1}$ and $p_{2}$ :

$$
p_{2} \geq p_{1} \geq \frac{\mu k}{\mu^{2}+\mu k-k^{2}}, \quad ; \quad \frac{\mu k}{\mu^{2}+\mu k-k^{2}} \geq p_{2} \geq p_{1}, \quad ; \quad p_{2} \geq \frac{\mu k}{\mu^{2}+\mu k-k^{2}} \geq p_{1} .
$$

Case I: First consider the demand scenario where $p_{2} \geq p_{1} \geq \frac{\mu k}{\mu^{2}+\mu k-k^{2}}$, the objective difference between the sequences is presented below for different intervals of supply.

\begin{tabular}{|c|c|}
\hline Interval of $s$ & $\mathcal{Z}_{1 \rightarrow 2}(s)-\mathcal{Z}_{2 \rightarrow 1}(s)$ \\
\hline$(0,2 \mu-k]$ & $s\left[\frac{1-p_{1}}{2} p_{2}-\frac{1-p_{2}}{2} p_{1}\right]\left[\frac{1}{2 \mu+k}+\frac{1}{2 \mu-k}-\frac{1}{2 \mu}-\frac{1}{2(\mu+k)}\right]$ \\
$(2 \mu-k, 2 \mu]$ & $s\left[\frac{1-p_{1}}{2} p_{2}-\frac{1-p_{2}}{2} p_{1}\right]\left[\frac{1}{2 \mu+k}+\frac{1}{s}-\frac{1}{2 \mu}-\frac{1}{2(\mu+k)}\right]$ \\
$(2 \mu, 2 \mu+k]$ & {$\left[\frac{1-p_{1}}{2} p_{2}-\frac{1-p_{2}}{2} p_{1}\right]\left[\frac{s}{2 \mu+k}-\frac{s-\mu}{\mu+k}\right]$} \\
$(2 \mu+k, \infty)$ & 0 \\
\hline
\end{tabular}

For all values of $s$ in this demand scenario, $\mathcal{Z}_{1 \rightarrow 2}(s)-\mathcal{Z}_{2 \rightarrow 1}(s)$ can be easily shown to be positive.

Case II: Now consider the demand scenario where $\frac{\mu k}{\mu^{2}+\mu k-k^{2}} \geq p_{2} \geq p_{1}$, the objective difference between the sequences is presented below for different intervals of supply.

\begin{tabular}{|c|c|}
\hline Interval of $s$ & $\mathcal{Z}_{1 \rightarrow 2}(s)-\mathcal{Z}_{2 \rightarrow 1}(s)$ \\
\hline$(0,2(\mu-k)]$ & {$\left[\frac{1-p_{1}}{2} p_{2}-\frac{1-p_{2}}{2} p_{1}\right]\left[\frac{s}{2 \mu+k}+\frac{s}{2 \mu-k}-\frac{s-\mu+k}{\mu}-\frac{s}{2(\mu+k)}\right]$} \\
$(2(\mu-k), 2 \mu-k]$ & $s\left[\frac{1-p_{1}}{2} p_{2}-\frac{1-p_{2}}{2} p_{1}\right]\left[\frac{1}{2 \mu+k}+\frac{1}{2 \mu-k}-\frac{1}{2 \mu}-\frac{1}{2(\mu+k)}\right]$ \\
$(2 \mu-k, 2 \mu]$ & $s\left[\frac{1-p_{1}}{2} p_{2}-\frac{1-p_{2}}{2} p_{1}\right]\left[\frac{1}{2 \mu+k}+\frac{1}{s}-\frac{1}{2 \mu}-\frac{1}{2(\mu+k)}\right]$ \\
$(2 \mu, 2 \mu+k]$ & {$\left[\frac{1-p_{1}}{2} p_{2}-\frac{1-p_{2}}{2} p_{1}\right]\left[\frac{s}{2 \mu+k}-\frac{s-\mu}{\mu+k}\right]$} \\
$(2 \mu+k, \infty)$ & 0 \\
\hline
\end{tabular}


For all values of $s$ in this demand scenario, $\mathcal{Z}_{1 \rightarrow 2}(s)-\mathcal{Z}_{2 \rightarrow 1}(s)$ can be easily shown to be positive.

Case III: Lastly consider the demand scenario where $p_{2} \geq \frac{\mu k}{\mu^{2}+\mu k-k^{2}} \geq p_{1}$. We have already shown that $\mathcal{Z}_{1 \rightarrow 2}(s)-\mathcal{Z}_{2 \rightarrow 1}(s) \geq 0$ for $p_{2}=\frac{\mu k}{\mu^{2}+\mu k-k^{2}} \geq p_{1}$. We show that $\frac{d\left(\mathcal{Z}_{1 \rightarrow 2}(s)-\mathcal{Z}_{2 \rightarrow 1}(s)\right)}{d p_{2}} \geq 0$, thus for $p_{2}>\frac{\mu k}{\mu^{2}+\mu k-k^{2}}$, then the difference remains positive.

For $s \leq 2(\mu-k)$, we have

$$
\frac{d\left(\mathcal{Z}_{1 \rightarrow 2}(s)-\mathcal{Z}_{2 \rightarrow 1}(s)\right)}{d p_{2}}=\frac{1}{2} \frac{k}{2(\mu+k)(2 \mu+k)}+\frac{1-p_{1}}{2}\left[\frac{k^{2}}{\mu(\mu-k)(\mu+k)}+\frac{\mu}{(2 \mu-k)(\mu+k)}\right]
$$

which is positive.

For $2 \mu-k \geq s \geq 2(\mu-k)$, we have:

$$
\begin{aligned}
\frac{d\left(\mathcal{Z}_{1 \rightarrow 2}(s)-\mathcal{Z}_{2 \rightarrow 1}(s)\right)}{d p_{2}}= & \frac{1-p_{1}}{2}\left[\frac{k(2 \mu-k-s)}{(2 \mu-k)(\mu+k)}+\frac{s k}{2(\mu+k)(2 \mu+k)}+\frac{s k}{2 \mu(2 \mu-k)}\right] \\
& +\frac{p_{1}}{2}\left[\frac{s k}{2(\mu+k)(2 \mu+k)}+\frac{s k+(s-2(\mu-k))(\mu+k)}{2 \mu(\mu+k)}\right]
\end{aligned}
$$

which is positive for $s$ in this interval.

For $s \geq 2 \mu-k$ the analysis is the same as in the other demand scenarios.

\section{Derivation of Optimal Policies for Proposition 1}

In what follows, we derive the optimal allocation policy for the problem in which nodes observe demand from distributions described in Figure $5(l e f t)$.

First consider the case where $d_{1}=\mu+k$. Let $x_{1}$ be the allocation to node 1 , and thus $s-x_{1}$ is the allocation to node 2 . The value of this allocation is:

$$
\mathcal{B}_{D_{2}}\left(x_{1} \mid s, 1, d_{1}=\mu+k\right)=\frac{1-p_{2}}{2}\left[\frac{x_{1}}{\mu+k} \wedge \frac{s-x_{1}}{\mu+k}\right]+p_{2}\left[\frac{x_{1}}{\mu+k} \wedge \frac{s-x_{1}}{\mu}\right]+\frac{1-p_{2}}{2}\left[\frac{x_{1}}{\mu+k} \wedge \frac{s-x_{1}}{\mu-k}\right] .
$$

To determine the optimal $x_{1}$, we consider the value of $\mathcal{B}_{D_{2}}\left(x_{1} \mid s, 1, d_{1}=\mu+k\right)$ for different intervals of $\frac{x_{1}}{\mu+k}$. If $\frac{x_{1}}{\mu+k} \leq \frac{s-x_{1}}{\mu+k} \leq \frac{s-x_{1}}{\mu} \leq \frac{s-x_{1}}{\mu-k}$, then

$$
\mathcal{B}_{D_{2}}\left(x_{1} \mid s, 1, d_{1}=\mu+k\right)=\frac{x_{1}}{\mu+k}
$$

and is increasing as $x_{1}$ increases. Therefore $\mathcal{B}_{D_{2}}\left(x_{1} \mid s, 1, d_{1}=\mu+k\right)$ is maximized in this interval at $x_{1}=s / 2$. If $\frac{s-x_{1}}{\mu+k} \leq \frac{x_{1}}{\mu+k} \leq \frac{s-x_{1}}{\mu} \leq \frac{s-x_{1}}{\mu-k}$, then

$$
\mathcal{B}_{D_{2}}\left(x_{1} \mid s, 1, d_{1}=\mu+k\right)=\frac{1-p_{1}}{2} \frac{s-x_{1}}{\mu+k}+\frac{1+p_{2}}{2} \frac{x_{1}}{\mu+k}
$$

and is increasing as $x_{1}$ increases. Therefore $\mathcal{B}_{D_{2}}\left(x_{1} \mid s, 1, d_{1}=\mu+k\right)$ is maximized in this interval at $x_{1}=s \frac{\mu+k}{2 \mu+k}$. If $\frac{s-x_{1}}{\mu+k} \leq \frac{s-x_{1}}{\mu} \leq \frac{x_{1}}{\mu+k} \leq \frac{s-x_{1}}{\mu-k}$, then

$$
\mathcal{B}_{D_{2}}\left(x_{1} \mid s, 1, d_{1}=\mu+k\right)=\frac{1-p_{1}}{2} \frac{s-x_{1}}{\mu+k}+p_{1} \frac{s-x_{1}}{\mu}+\frac{1-p_{2}}{2} \frac{x_{1}}{\mu+k}
$$

and is increasing as $x_{1}$ decreases. Therefore $\mathcal{B}_{D_{2}}\left(x_{1} \mid s, 1, d_{1}=\mu+k\right)$ is maximized in this interval at $x_{1}=s \frac{\mu+k}{2 \mu+k}$. If $\frac{s-x_{1}}{\mu+k} \leq \frac{s-x_{1}}{\mu} \leq \frac{s-x_{1}}{\mu-k} \leq \frac{x_{1}}{\mu+k}$ then

$$
\mathcal{B}_{D_{2}}\left(x_{1} \mid s, 1, d_{1}=\mu+k\right)=\frac{1-p_{1}}{2} \frac{s-x_{1}}{\mu+k}+p_{1} \frac{s-x_{1}}{\mu}+\frac{1-p_{2}}{2} \frac{s-x_{1}}{\mu+k}
$$


and is increasing as $x_{1}$ decreases. Therefore $\mathcal{B}_{D_{2}}\left(x_{1} \mid s, 1, d_{1}=\mu+k\right)$ is maximized in this interval at $x_{1}=s \frac{\mu+k}{2 \mu}$. From this interval analysis, we conclude that $\mathcal{B}_{D_{2}}\left(x_{1} \mid s, 1, d_{1}=\mu+k\right)$ is maximized at $s \frac{\mu+k}{2 \mu+k}$. Since $x_{1} \leq d_{1}$, then $x_{1}^{*}=(\mu+k) \wedge s \frac{\mu+k}{2 \mu+k}$.

Now consider the case where $d_{1}=\mu$. The value of this allocation is:

$$
\mathcal{B}_{D_{2}}\left(x_{1} \mid s, 1, d_{1}=\mu\right)=\frac{1-p_{2}}{2}\left[\frac{x_{1}}{\mu} \wedge \frac{s-x_{1}}{\mu+k}\right]+p_{2}\left[\frac{x_{1}}{\mu} \wedge \frac{s-x_{1}}{\mu}\right]+\frac{1-p_{2}}{2}\left[\frac{x_{1}}{\mu} \wedge \frac{s-x_{1}}{\mu-k}\right] .
$$

We consider the value of $\mathcal{B}_{D_{2}}\left(x_{1} \mid s, 1, d_{1}=\mu\right)$ for different intervals of $\frac{x_{1}}{\mu}$.

If $\frac{x_{1}}{\mu} \leq \frac{s-x_{1}}{\mu+k} \leq \frac{s-x_{1}}{\mu} \leq \frac{s-x_{1}}{\mu-k}$, then

$$
\mathcal{B}_{D_{2}}\left(x_{1} \mid s, 1, d_{1}=\mu\right)=\frac{x_{1}}{\mu}
$$

and is increasing as $x_{1}$ increases. Therefore $\mathcal{B}_{D_{2}}\left(x_{1} \mid s, 1, d_{1}=\mu\right)$ is maximized in this interval at $x_{1}=s \frac{\mu}{2 \mu+k}$.

If $\frac{s-x_{1}}{\mu+k} \leq \frac{x_{1}}{\mu} \leq \frac{s-x_{1}}{\mu} \leq \frac{s-x_{1}}{\mu-k}$, then

$$
\mathcal{B}_{D_{2}}\left(x_{1} \mid s, 1, d_{1}=\mu\right)=\frac{1-p_{1}}{2} \frac{s-x_{1}}{\mu+k}+\frac{1+p_{2}}{2} \frac{x_{1}}{\mu}
$$

and is increasing as $x_{1}$ increases. Therefore $\mathcal{B}_{D_{2}}\left(x_{1} \mid s, 1, d_{1}=\mu\right)$ is maximized in this interval at $x_{1}=s / 2$.

If $\frac{s-x_{1}}{\mu+k} \leq \frac{s-x_{1}}{\mu} \leq \frac{x_{1}}{\mu} \leq \frac{s-x_{1}}{\mu-k}$, then

$$
\mathcal{B}_{D_{2}}\left(x_{1} \mid s, 1, d_{1}=\mu\right)=\frac{1-p_{1}}{2} \frac{s-x_{1}}{\mu+k}+p_{2} \frac{s-x_{1}}{\mu}+\frac{1-p_{2}}{2} \frac{x_{1}}{\mu}
$$

and is increasing as $x_{1}$ decreases. Therefore $\mathcal{B}_{D_{2}}\left(x_{1} \mid s, 1, d_{1}=\mu\right)$ is maximized in this interval at $x_{1}=s / 2$.

If $\frac{s-x_{1}}{\mu+k} \leq \frac{s-x_{1}}{\mu} \leq \frac{s-x_{1}}{\mu-k} \leq \frac{x_{1}}{\mu}$, then

$$
\mathcal{B}_{D_{2}}\left(x_{1} \mid s, 1, d_{1}=\mu\right)=\frac{1-p_{1}}{2} \frac{s-x_{1}}{\mu+k}+p_{2} \frac{s-x_{1}}{\mu}+\frac{1-p_{2}}{2} \frac{s-x_{1}}{\mu-k}
$$

and is increasing as $x_{1}$ decreases. Therefore $\mathcal{B}_{D_{2}}\left(x_{1} \mid s, 1, d_{1}=\mu\right)$ is maximized in this interval at $x_{1}=s \frac{\mu}{2 \mu-k}$.

From this interval analysis, we conclude that $\mathcal{B}_{D_{2}}\left(x_{1} \mid s, 1, d_{1}=\mu\right)$ is maximized at $s / 2$. Since $x_{1} \leq d_{1}$, then $x_{1}^{*}=\mu \wedge s / 2$.

Lastly, consider the case where $d_{1}=\mu-k$. The value of this allocation is:

$$
\mathcal{B}_{D_{2}}\left(x_{1} \mid s, 1, d_{1}=\mu-k\right)=\frac{1-p_{2}}{2}\left[\frac{x_{1}}{\mu-k} \wedge \frac{s-x_{1}}{\mu+k}\right]+p_{2}\left[\frac{x_{1}}{\mu-k} \wedge \frac{s-x_{1}}{\mu}\right]+\frac{1-p_{2}}{2}\left[\frac{x_{1}}{\mu-k} \wedge \frac{s-x_{1}}{\mu-k}\right] .
$$

We consider the value of $\mathcal{B}_{D_{2}}\left(x_{1} \mid s, 1, d_{1}=\mu-k\right)$ for different intervals of $\frac{x_{1}}{\mu-k}$.

If $\frac{x_{1}}{\mu-k} \leq \frac{s-x_{1}}{\mu+k} \leq \frac{s-x_{1}}{\mu} \leq \frac{s-x_{1}}{\mu-k}$, then

$$
\mathcal{B}_{D_{2}}\left(x_{1} \mid s, 1, d_{1}=\mu-k\right)=\frac{x_{1}}{\mu-k}
$$

and is increasing as $x_{1}$ increases. Therefore $\mathcal{B}_{D_{2}}\left(x_{1} \mid s, 1, d_{1}=\mu-k\right)$ is maximized in this interval at $x_{1}=s \frac{\mu-k}{2 \mu}$. If $\frac{s-x_{1}}{\mu+k} \leq \frac{x_{1}}{\mu-k} \leq \frac{s-x_{1}}{\mu} \leq \frac{s-x_{1}}{\mu-k}$, then

$$
\mathcal{B}_{D_{2}}\left(x_{1} \mid s, 1, d_{1}=\mu-k\right)=\frac{1-p_{2}}{2} \frac{s-x_{1}}{\mu+k}+\frac{1+p_{2}}{2} \frac{x_{1}}{\mu-k}
$$


and is increasing as $x_{1}$ increases. Therefore $\mathcal{B}_{D_{2}}\left(x_{1} \mid s, 1, d_{1}=\mu-k\right)$ is maximized in this interval at $x_{1}=s \frac{\mu-k}{2 \mu-k}$. If $\frac{s-x_{1}}{\mu+k} \leq \frac{s-x_{1}}{\mu} \leq \frac{x_{1}}{\mu-k} \leq \frac{s-x_{1}}{\mu-k}$, then

$$
\mathcal{B}_{D_{2}}\left(x_{1} \mid s, 1, d_{1}=\mu-k\right)=\frac{1-p_{2}}{2} \frac{s-x_{1}}{\mu+k}+p_{2} \frac{s-x_{1}}{\mu}+\frac{1-p_{2}}{2} \frac{x_{1}}{\mu-k}
$$

and is increasing as $x_{1}$ increases if $p_{2}<\frac{\mu k}{\mu^{2}+\mu k-k^{2}}$, otherwise it is increasing as $x_{1}$ decreases. Therefore $\mathcal{B}_{D_{2}}\left(x_{1} \mid s, 1, d_{1}=\mu-k\right)$ is maximized in this interval at $x_{1}=s / 2$ if $p_{2}<\frac{\mu k}{\mu^{2}+\mu k-k^{2}}$ and $x_{1}=s \frac{\mu-k}{2 \mu-k}$ otherwise.

If $\frac{s-x_{1}}{\mu+k} \leq \frac{s-x_{1}}{\mu} \leq \frac{s-x_{1}}{\mu-k} \leq \frac{x_{1}}{\mu-k}$, then

$$
\mathcal{B}_{D_{2}}\left(x_{1} \mid s, 1, d_{1}=\mu-k\right)=\frac{1-p_{2}}{2} \frac{s-x_{1}}{\mu+k}+p_{2} \frac{s-x_{1}}{\mu}+\frac{1-p_{2}}{2} \frac{s-x_{1}}{\mu-k}
$$

and is increasing as $x_{1}$ decreases. Therefore $\mathcal{B}_{D_{2}}\left(x_{1} \mid s, 1, d_{1}=\mu-k\right)$ is maximized in this interval at $x_{1}=s / 2$.

From this interval analysis, we conclude that $\mathcal{B}_{D_{2}}\left(x_{1} \mid s, 1, d_{1}=\mu-k\right)$ is maximized at $s / 2$ if $p_{2}<\frac{\mu k}{\mu^{2}+\mu k-k^{2}}$ and at $s \frac{\mu-k}{2 \mu-k}$ otherwise. Since $x_{1} \leq d_{1}$, then if $p_{2}<\frac{\mu k}{\mu^{2}+\mu k-k^{2}}, x_{1}^{*}=\mu-k \wedge s / 2$ and if $p_{2} \geq \frac{\mu k}{\mu^{2}+\mu k-k^{2}}$, $x_{1}^{*}=\mu-k \wedge s \frac{\mu-k}{2 \mu-k}$. 